Publications of the Astronomical Society of the Pacific, 117:173-188, 2005 February

(C) 2005. The Astronomical Society of the Pacific. All rights reserved. Printed in U.S.A.

\title{
The Prospects for Finding Brown Dwarfs in Eclipsing Binary Systems and Measuring Brown Dwarf Properties
}

\author{
D. J. Pinfield, H. R. A. Jones, And I. A. Steele \\ Astrophysics Research Institute, Liverpool John Moores University, 12 Quays House, Egerton Wharf, Birkenhead CH41 1LD, UK; dpi@star.herts.ac.uk \\ Received 2004 July 2; accepted 2004 December 5; published 2005 February 9
}

\begin{abstract}
We present the results of a simulation to investigate the prospects of measuring mass, age, radius, metallicity, and luminosity data for brown dwarfs in fully eclipsing binary systems around dwarf spectral types from late $\mathrm{K}$ to early $\mathrm{M}$ that could be identified by ultra-wide-field transit surveys such as SuperWASP. These surveys will monitor approximately a million $\mathrm{K}$ and $\mathrm{M}$ dwarfs with $|b|>20^{\circ}$ (where blending of sources is not a significant problem) at a level sufficient to detect transits of low-luminosity companions. We look at the current observational evidence for such systems and suggest that $\sim 1 \%$ of late $\mathrm{K}$ and early-to-mid $\mathrm{M}$ dwarfs could have a very close ( 0.02 AU) brown dwarf companion. With this assumption, and using SuperWASP as an example, our simulation predicts that $\sim 400$ brown dwarfs in fully eclipsing binary systems could be discovered. All of these eclipsing binaries could yield accurate brown dwarf mass and radius measurements from radial velocity and follow-up light curve measurements. By inferring the brown dwarf effective temperature distribution, assuming a uniform age spread and an $\alpha=0.5$ companion brown dwarf mass function, the simulation estimates that brown dwarf brightness could also be measurable (at the 10\% level) for $\sim 60$ of these binary systems from near-infrared follow-up light curves of the secondary eclipse. We consider irradiation of these brown dwarfs by their primary stars and conclude that it would be below the $10 \%$ level for $\sim 70 \%$ of them. This means that in these cases, the measured brown dwarf brightnesses should essentially be the same as those of free-floating counterparts. The predicted age distribution of the primaries is dominated by young systems, and $\sim 20$ binaries could be younger than 1 Gyr. Irradiation will be below the $10 \%$ level for $\sim 80 \%$ of these. We suggest that many of these young binary systems will be members of "kinematic moving groups," allowing their ages to be accurately constrained.
\end{abstract}

\section{INTRODUCTION}

The mass of a brown dwarf (BD) is arguably its most basic characteristic. The essence of the classification of an object as a BD depends on its ability to sustain deuterium burning (for solar metallicity, $>0.013 M_{\odot}$ ) but not hydrogen burning (for solar metallicity, $<0.075 M_{\odot}$ ). However, since BDs contract, cool, and fade with time, their observable properties depend strongly on both age and mass. Furthermore, because BD colors are strongly influenced by atmospheric dust formation and molecular absorbers (such as $\mathrm{H}_{2} \mathrm{O}$ ), their appearance will depend not only on effective temperature $\left(T_{\text {eff }}\right)$ and surface gravity $(g)$, but also on composition. Accurate BD mass, age, radius, metallicity, and luminosity data are thus vital to properly test our understanding of these extreme objects.

In view of the particular importance of such data for BDs, it is unfortunate that it has thus far not been possible to completely characterize any BDs in this way. There are several reasons for this. In order to measure BD masses, one must find BDs in binary systems and measure the orbits. One also needs to be able to measure the BD itself to determine its luminosity, and the age of the binary must be established, so as to infer the age of the BD. Proper motion measurements have shown that $\sim 10 \%$ of solar-type stars have BD companions at wide (>1000 AU) separations (Gizis et al. 2001). However, the periods of such binaries are much too long to be useful in measuring masses. A small number of possible close BD companions to solar-type stars have been identified (Halbwachs et al. 2000; Nidever et al. 2002; Blundell et al. 2003). But such systems are very rare and could not be directly imaged anyway, because of the glare of the primary. For the lowest mass stars, adaptive optics imaging has shown that close ( 1 AU) BD companions to late $\mathrm{M}$ and early $\mathrm{L}$ dwarfs are significantly more common (Close et al. 2003), with orbits that can be measured in a few years, and binary components that can be individually studied. For example, Bouy et al. (2004) astrometrically measured $36 \%$ of the $10 \mathrm{yr}$ orbit of $2 \mathrm{MASS}$ J0746 $+2000 \mathrm{AB}$, constraining the system mass at the $10 \%$ level. However, the ages of such binaries will in general be very difficult to constrain without recourse to the theoretical models that one wishes to test. For instance, Bouy et al. compared 2MASS J0746+2000 A and B with "dusty model" predictions (Chabrier et al. 2000) in a $(K, J-K)$ color-magnitude diagram (CMD) and estimated a system age of 150-500 Myr. However, when compared with late $\mathrm{M}$ and $\mathrm{L}$ dwarfs with accurate parallaxes, 2MASS 
TABLE 1

Brown Dwarf Companion Fractions as a Function of Primary Type

\begin{tabular}{|c|c|c|c|c|c|c|c|}
\hline \multirow[b]{2}{*}{ Primary Spectral Type } & \multicolumn{7}{|c|}{ SEPARATION (AU) } \\
\hline & $0.001-0.01$ & $0.01-0.1$ & $0.1-1$ & $1-10$ & $10-100$ & $100-1000$ & $1000-10,000$ \\
\hline \multicolumn{8}{|l|}{ Stellar Companions: } \\
\hline $\mathrm{F}, \mathrm{G}$, and $\mathrm{K} \ldots \ldots \ldots$ & a & \multicolumn{3}{|c|}{$\sim 4 \%$ per decade $(1)$} & \multicolumn{3}{|c|}{$\sim 10 \%-15 \%$ per decade $(2)$} \\
\hline \multicolumn{8}{|l|}{ Brown Dwarf Companions: } \\
\hline $\mathrm{F}, \mathrm{G}$, and $\mathrm{K} \ldots \ldots \ldots$ & a & \multicolumn{3}{|c|}{$<0.07 \%$ per decade $(3)$} & $?(4)$ & $\sim 10 \%(5)$ & $5 \%-13 \%(6)$ \\
\hline Early to mid M & $0 \%-2 \%(7)$ & $?$ & $1 \%(8)$ & $1 \%(8)$ & $1 \%-3 \%(9)$ & $\ldots$ & $\ldots$ \\
\hline Late $\mathrm{M}, \mathrm{L}$, and $\mathrm{T} \ldots \ldots$ & & $\leq 30 \%(10)$ & & $10 \%-20 \%(11)$ & $\ldots$ & $\ldots$ & $\ldots$ \\
\hline
\end{tabular}

Note. - The BD companion fraction around early-to-mid M dwarfs for $a \leq 0.02 \mathrm{AU}$ is $0 \%-2 \%$.

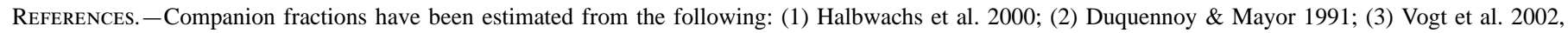

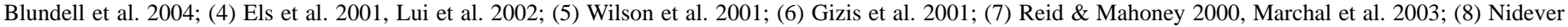
et al. 2002; (9) Oppenheimer et al. 2001; (10) Reiners 2004, Pinfield et al. 2003; (11) Burgasser et al. 2003, Close et al. 2003 a, Reid et al. 2001.

${ }^{\text {a }}$ Not applicable $\left(R_{\odot}=0.005 \mathrm{AU}\right)$.

J0746+2000 A and B do not lie above the CMD sequence, as sources of this age should. A potentially more useful type of system is represented by the $\epsilon$ Indi multiple, which consists of a double-BD binary (visual separation of $2.65 \mathrm{AU}$ ) at a distance of 1500 AU from its K5 primary (McCaughrean et al. 2004). The BD binary orbit should be measurable $(P \sim 15 \mathrm{yr})$, and the age of the system can be constrained to some degree (0.8-2 Gyr) by measurements of the $\mathrm{K}$ dwarf primary (Lachaume et al. 1999). However, such multiple systems are not commonplace, and age constraints established from measurements of a single primary star will not generally be very precise.

The concept presented here is that of exploiting the new breed of ultra-wide-field transit surveys (designed primarily to find signatures of planetary transits) to identify BDs in fully eclipsing close binary systems around cool stars. BD masses could be measured from the Doppler wobble of the primary (using radial velocity techniques). One could measure BD radii from the depth of the primary eclipse (when the BD crosses the face of the primary). The brightness of the BD could be measured from the secondary eclipse (when the BD goes behind the primary), provided the BD is bright enough compared to the primary. Many of these systems will be young (because BDs are brighter when young), making membership in young kinematic moving groups more likely. Membership in such moving groups would accurately constrain age and metallicity.

In this paper, we discuss the prospects of identifying BDs in binaries capable of providing the full compliment of BD mass, age, radius, metallicity, and luminosity data. In $\S 2$ we discuss what is currently known about the frequency and separation of close BD companions, and how this effects the likelihood of eclipse. In $\S 3$ we use the SuperWASP survey as an example of the new ultra-wide-field transit surveys, estimate sensitivities and the effects of source crowding in images with very large pixels, and estimate the distances out to which BD transits should be detectable. Section 4 describes the additional factors limiting the number of suitable binary systems: the accuracy with which secondary eclipses can be measured $(\S 4.1)$ and the limits imposed by the Doppler wobble technique ( $\$ 4.2$ ). Section 5 gives some background on kinematic moving groups and details how one could accurately constrain the ages of young systems. Section 6 describes our simulation to predict the size and properties of the eclipsing BD population. Section 7 then discusses some identification and follow-up procedures, and $\S 8$ presents our conclusions.

\section{CLOSE BROWN DWARF COMPANIONS}

\subsection{Frequency and Separation}

In Table 1 we show and compare what is known about the frequency of companion stars and BDs as a function of separation. For solar-type stars, Halbwachs et al. (2003) and Duquennoy \& Mayor (1991) have measured the frequency of stellar companions as a function of period (which we convert into separation) using $10 \mathrm{yr}$ of radial velocity data combined with visual binaries and common proper motion systems. It is clear from this work that stellar companions are common over a large range of separation. However, it has been known for some time that there is a dearth of low mass ratio $(q \leq 0.1)$ companions in close orbits around solar-type stars, known as the "BD desert." Other radial velocity surveys (e.g., Marcy \& Benitz 1989; Fischer \& Marcy 1992; Nidever et al. 2002) have now studied large numbers of solar-type stars, and it is currently estimated that $\leq 1 / 500$ have a BD companion within $3 \mathrm{AU}$ (Vogt et al. 2002). This corresponds to a BD companion fraction of less than $0.07 \%$ per decade in separation (clearly much less than the $\sim 4 \%$ for stellar companions). The desert does not extend to wide separations, with substellar companions imaged at 15-20 AU (Els et al. 2001; Lui et al. 2002), and BDs are as common in very wide binaries ( 1000 AU or more) as stellar companions around solar-type stars.

In terms of primary type, there is evidence (from the OGLE transit survey) that the desert extends out to the mid $\mathrm{K}$ dwarfs, since the majority of the small, low-luminosity companions discovered by Udalski et al. (2002a, 2002b, 2003) turned out to be low-mass stars and not BDs (Dreizler et al. 2002). However, for later dwarfs the target lists for radial velocity surveys 


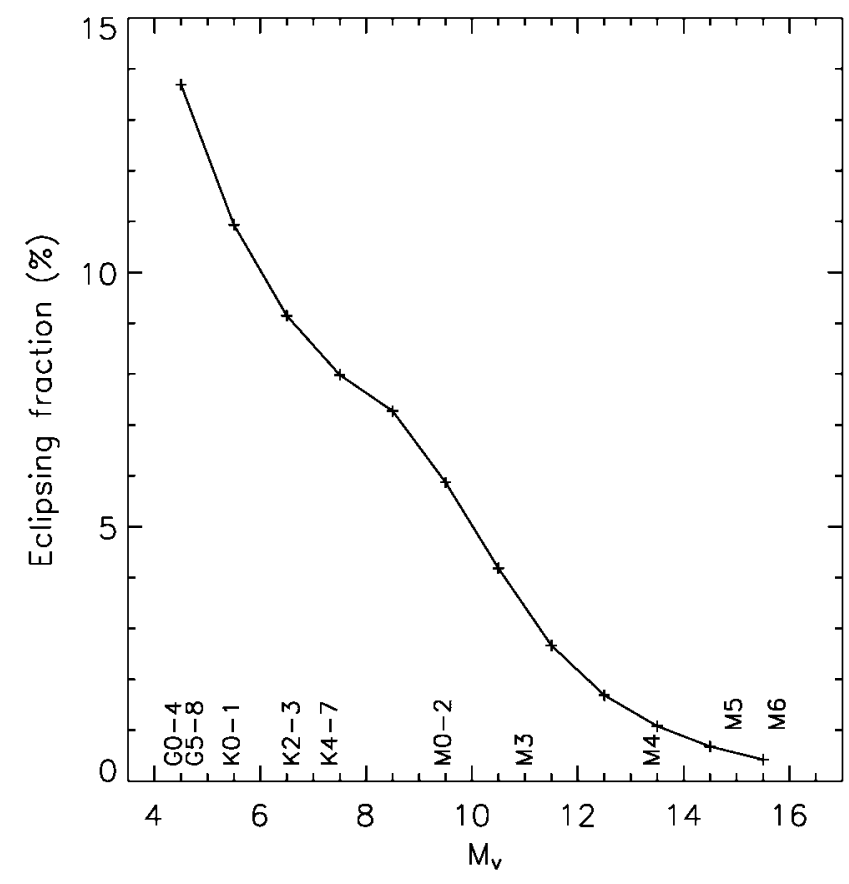

FIG. 1.-Fraction of companion BDs $\left(0.1 R_{\odot}\right)$ that will fully eclipse their primary star at a separation of $0.02 \mathrm{AU}$ vs. primary type.

are not large enough to accurately constrain the close BD companion fraction. Despite these limitations, some interesting candidate BD companions have been discovered. Reid \& Mahoney (2000) found a possible BD companion $\left(0.07-0.095 M_{\odot}\right)$ at a separation of $\sim 0.005-0.007$ AU from an $\sim$ M5 dwarf in a radial velocity survey of $\sim 50$ Hyades M dwarfs. Nidever et al. (2002) found two possible BD companions (at separations of $\sim 0.2$ and $\sim 2 \mathrm{AU}$ ) around early $\mathrm{M}$ dwarfs from a radial velocity survey including $\sim 100 \mathrm{M}$ dwarfs. Conversely, Marchal et al. (2003) found no close BD companions in a comparable radial velocity survey of $\sim 100$ M0-M5 dwarfs.

At somewhat larger separations, Oppenheimer et al. (2001) found one $\mathrm{BD}$ in a coronagraphic survey of the stellar population within $8 \mathrm{pc}$ of the Sun. This volume-limited sample (i.e., mostly M dwarfs) consisted of 163 stars in 111 star systems, and BDs with masses $\geq 0.04 M_{\odot}$ and ages $\leq 5$ Gyr could have been detected with separations of 40-120 AU in around 80\% of the sample. BDs are not found in significantly wider orbits around early $\mathrm{M}$ dwarfs, perhaps because impulsive perturbations by close stellar encounters will cause such weakly bound systems to dissolve (see Burgasser et al. 2003). It is not, then, surprising that this lack of wide BD companions is also seen around the latest stars and BDs (with the possible exception of one very young candidate BD binary, which would have a separation of 240 AU; Luhman 2004). However, closer companions to late M, L, and T dwarfs are quite common (see Table 2), and while accurate masses are generally not available, many of these will be substellar (e.g., an L7.5 BD 3 AU from an M8 star; Freed et al. 2003).

Of interest here are the late $\mathrm{K}$ to mid $\mathrm{M}$ dwarf primaries, since we subsequently show that sufficient numbers of these dwarfs will be monitored by ultra-wide-field surveys to find eclipsing BD companions (see $\S 6$ ). It is clear from Table 1 that BD companions to these stars could occur at the $\sim 2 \%$ level in orbits close enough to make transits likely $(\leq 0.02 \mathrm{AU}$; see $\S 2.2$ ). However, an accurate determination of the masses of the BD candidates discovered so far would require knowledge of orbital inclination. Some may turn out to be low-mass stars at low inclination (see, e.g., Halbwachs et al. 2000). If correct, though, it would show that although the BD companion fraction in close orbits around such stars is lower than that of stellar companions, there would be no BD desert for these primaries.

In addition to these systematic searches, there have been two serendipitous discoveries of possible close BD companions. Schuh et al. (2003) reported the discovery of an eclipsing BD companion to a late $\mathrm{K}$ dwarf with a separation of $0.02 \mathrm{AU}$. This system was discovered while photometrically monitoring a white dwarf. In addition, Santos et al. (2002) discovered a radial velocity $\mathrm{BD}$ companion to an $\mathrm{M} 2$ dwarf with a separation of $0.017 \mathrm{AU}$. This system was found as a result of the weak gravitational wobble it induced in the visual $\mathrm{K} 2$ companion of the $\mathrm{M}$ dwarf.

Having considered all known candidates, it can be seen that the spectral types of the primaries in systems that could potentially harbor significant populations of very close BD companions range from late $\mathrm{K}$ to mid $\mathrm{M}$. The orbital periods of these $\mathrm{BD}$ companions in close orbits around late $\mathrm{K}$ to mid $\mathrm{M}$ dwarfs is 1-1.5 days. At these separations, a full transit lasts $\sim 1.5 \mathrm{hr}$.

\subsection{Eclipsing Fraction}

The majority of the known candidate BD companions around $\mathrm{K}$ and $\mathrm{M}$ dwarfs (three out of five) are in close orbits with separations $\leq 0.02 \mathrm{AU}$ (see $\S 2.1$ ). Close systems such as these will be the most likely to eclipse, because eclipsing probability is $\propto 1 / a$ (where $a$ is separation). We therefore chose to consider the likelihood of an eclipse for a BD companion at a separation of $0.02 \mathrm{AU}$. This separation corresponds to $\sim 10 R_{*}$ for an early M dwarf, and for such separations, a favorable $\sim 1$ in 15 should be eclipsing. The requirement for a full eclipse is that

$$
\sin \left(90^{\circ}-i\right) \leq\left(R_{p}-R_{\mathrm{BD}}\right) / a,
$$

where $i$ is the inclination angle, $R_{p}$ and $R_{\mathrm{BD}}$ are the primary and $\mathrm{BD}$ radii, respectively, and $a$ is the component separation. The fraction of sources that should be eclipsing is $\left(90^{\circ}-i\right) / 90^{\circ}$. Using theoretical stellar radii from the 500 Myr NextGen models of Baraffe et al. (1998), and assuming $0.1 R_{\odot}$ for the BDs (typical for $500 \mathrm{Myr}$ age), we get the eclipse fractions shown in Figure 1. As the primary radius decreases, the chances of a 
full eclipse fall off, and for comparable radii, full eclipses are very unlikely. It can be seen that for late $\mathrm{K}$ to early $\mathrm{M}$ dwarfs, the eclipsing fraction is $\sim 4 \%-7 \%$, and so even with a $\sim 1 \%$ binary fraction at $0.02 \mathrm{AU}$, one could still expect to find fully eclipsing BD companions around $\sim 1$ in 2000 dwarfs of this type.

\subsection{Irradiation}

Since we are interested in measuring companion BD properties that would be essentially the same as those of free-floating counterparts, we desire irradiation effects to be low. However, for the very close BD companions discussed in $\S 2.2$, this may not always be the case, since the cool BD companion could be heated by the nearby brighter, hotter primary, as happens with giant planets in close orbits around solar-type stars (hot Jupiters). Hot Jupiters are strongly irradiated and have higher $T_{\text {eff }}$ 's and swollen radii as a result (e.g., Burrows et al. 2003; Chabrier et al. 2004). Irradiation should only be an issue when the incident flux from the primary is significant compared to the emergent flux from the companion. If the incident flux is $10 \%$ of the emergent flux, for instance, then the BD luminosity would increase by no more than $10 \%$ (and the radius and $T_{\text {eff }}$ by much less than this). This represents a useful level of accuracy with which to measure BD brightness. Figure 2 shows how the amount of irradiation varies for BD companions (spectral types M6-T4) at 0.02 AU separation from their primary stars. Each point in the plotting area represents a binary for which the primary and secondary spectral types can be read off the $y$ - and $x$-axis, respectively. Using NextGen model data for the primaries, BD $T_{\text {eff }}$ 's appropriate to their spectral type (Reid et al. 1999; Knapp et al. 2004), and assumed BD radii of $0.1 R_{\odot}$, we derived a locus of binary systems in which the BD will be irradiated by $10 \%$ of its emergent flux. This locus is plotted in the figure as a solid line, where binaries above the line are not significantly irradiated. For example, an M8 BD will not be significantly irradiated at $0.02 \mathrm{AU}$ from a mid $\mathrm{K}$ dwarf (or later) primary, and an L4 BD will not be significantly irradiated at 0.02 AU from an M0-M2 (or later) primary.

\subsection{Age and Mass Distributions}

The mass and age distribution of the companion BDs is important, because the older and lower mass BDs will be more difficult to measure from the secondary eclipse. The age distribution should be the same as the local disk. An analysis of chromospheric activity of $\mathrm{G}$ and $\mathrm{M}$ dwarfs in the solar vicinity (Soderblom et al. 1991; Henry et al. 1996) is consistent with a uniform star formation rate over the age of the disk, and we thus assume that BDs will have a uniform age distribution between 0 and 10 Gyr. The mass distribution of companion BDs is less constrained. The BD mass function slope for the field and young clusters and associations ranges from $\alpha \sim 0.5$ to $\sim 1$ (where $d n / d m \propto m^{-\alpha}$ ). However, the masses of BDs in close binaries may have a very different distribution, since dynamical

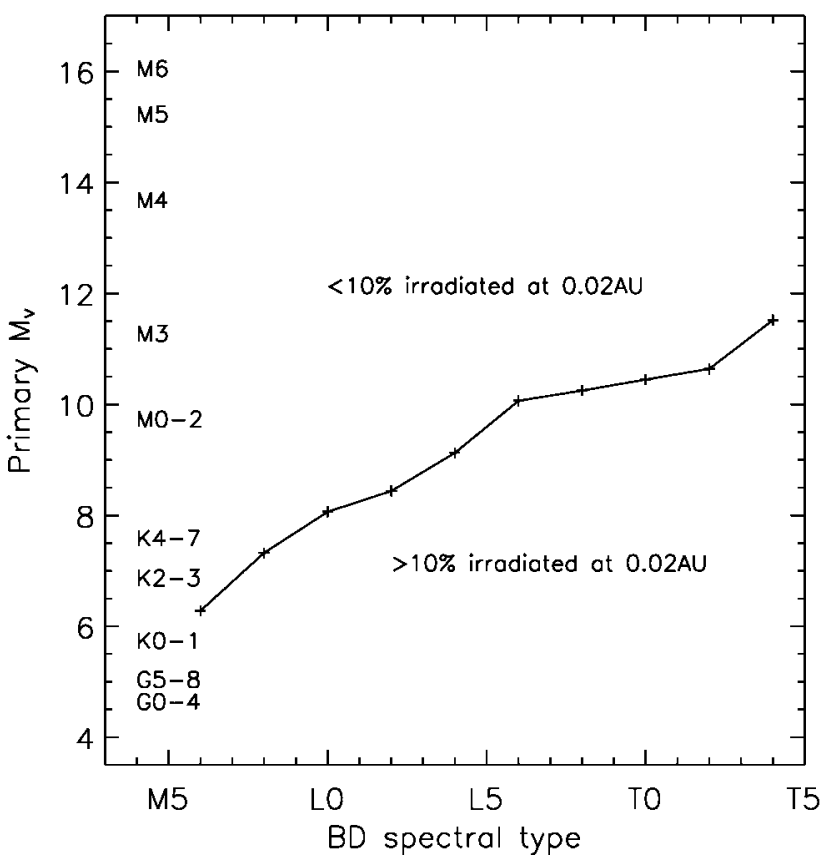

FIG. 2.-Primary $M_{V}$ vs. companion BD spectral type. The solid line represents binary systems in which the BDs are irradiated by $10 \%$ at a separation of $0.02 \mathrm{AU}$. The regions above and below the line represent lower and higher levels of BD irradiation, respectively.

factors during formation (e.g., Reipurth \& Clarke 2001) and possible radial migration (due to early disk interaction; Armitage \& Bonnell 2002) could be important. For very low mass binary systems, for instance, the $q$-distribution peaks at $0.7-1$ (Burgasser et al. 2003; Pinfield et al. 2003). Such a bias among binaries with an early $\mathrm{M}$ dwarf primary would produce a strong preference for stellar companions over BDs, and the BD companion mass function itself would prefer high-mass BDs. However, it is not currently possible to estimate $\alpha$ for close BD companions from the limited observational evidence, and it essentially remains a free parameter. Low values of $\alpha$ would result in proportionally more high-mass BDs (for some value of the BD companion fraction), which would be brighter and easier to detect. Higher values would mean proportionally more low-mass BDs, many of which would be too faint to detect.

\section{ULTRA-WIDE-FIELD TRANSIT SURVEYS}

The discovery of the first transiting extrasolar planet (Charbonneau et al. 2000; Henry et al. 2000b) has led to a proliferation of ground-based photometric searches aiming to detect planets around bright solar-type stars by searching for transit signatures in photometric light curves (see Horne 2003). These searches fall into two categories: the deeper but relatively small area searches such as the Optical Gravitational Lensing Experiment (OGLE, which uses a $1.3 \mathrm{~m}$ aperture telescope), and the ultra-wide-field surveys such as SuperWASP (Christian et al. 2004), TrES (Alonso et al. 2004), Vulcan South (Caldwell 


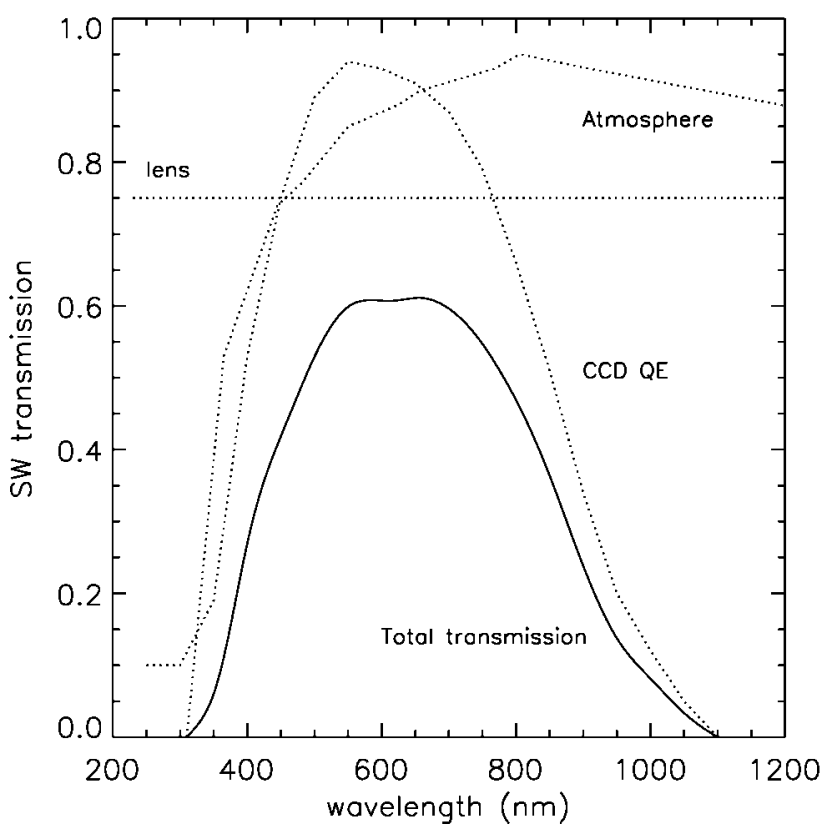

FIG. 3.-Transmission of SuperWASP. Atmospheric transmission (air mass $=1.5$ ), optical throughput, and CCD quantum efficiency are shown with dotted lines. The total transmission is shown with a solid line.

et al. 2004), and others, which use small-aperture $(\sim 10 \mathrm{~cm})$ wide-field $\left(5^{\circ}-10^{\circ}\right) \mathrm{CCD}$-based systems with a spatial resolution of $\sim 10^{\prime \prime}$ pixel $^{-1}$. While accurate light curve extraction is more challenging for ultra-wide-field data, the ultra-wide-field surveys promise to discover transits around brighter nearer stars, for which close transiting companions can be more accurately measured. In this paper, we focus on the abilities of these ultra-wide-field surveys and use the UK's SuperWASP project as a specific example.

\subsection{SuperWASP}

SuperWASP (Super Wide Angle Search for Planets) is a project to construct and operate facilities to carry out ultrawide-field unfiltered photometric monitoring surveys. ${ }^{1}$ The first SuperWASP facility (SuperWASP-I) is located on Roque de Los Muchachos, La Palma, and a second facility has also been funded for the Southern Hemisphere. SuperWASP-I consists of five camera units mounted on a large computer-controlled fork mounting, housed in a small enclosure. Each camera unit consists of a $200 \mathrm{~mm} \mathrm{f} / 1.8$ Canon lens with an aperture of $11.1 \mathrm{~cm}$ (made of fluorite and UD glass components) and a $2048 \times 2048$ pixel e2v42 thinned CCD ( $B V$ coated), cooled to $-60^{\circ} \mathrm{C}$ by a threestage Peltier. Each camera has an $8^{\circ} \times 8^{\circ}$ field of view, resulting in large, 14.3 CCD pixels. The combined sky coverage of the five cameras is thus $\sim 320 \mathrm{deg}^{2}$. The current observing strategy for SuperWASP-I is to repeatedly measure $30 \mathrm{~s}$ integration im-

\footnotetext{
${ }^{1}$ See Pollacco et al. at http://www.superwasp.org.
}

ages of the same region of sky throughout each night for a period of $\sim 2$ months, before moving onto a new region. With low overheads ( $\sim 4$ s readout), SuperWASP-I takes $\sim 100$ sets of images per hour. First light was in 2003 November, and at the time of writing, SuperWASP-I is routinely taking data. A data reduction pipeline is being implemented, and light curves of all stars measured will be placed on the public "LEDAS database" at the University of Leicester, where software search algorithms will be available for data mining.

\subsection{SuperWASP Sensitivities}

SuperWASP is an unfiltered system, and as such the transmission profile (or passband) is determined by the atmosphere, the optical throughput of the lenses and CCD entrance window, and the CCD quantum efficiency. To estimate the shape of the SuperWASP transmission profile, we have used available site and technical details. We used La Palma atmospheric extinctions from the INT Wide Field Survey Web site (based on observations by Derek Jones in 1993 August). For the Canon lenses, the optical transmission of UD glass and fluorite ( $95 \%$ per pair of air-glass interfaces) does not vary significantly from $\sim 0.23$ to $7 \mu \mathrm{m}$ (minimizing chromospheric aberration). We thus assumed a wavelength-independent optical transmission of $75 \%$ to allow for the lens groups (in each Canon lens) and the CCD entrance window. The CCD quantum efficiency was taken from the E2V CCD selection guide, interpolating between the $-20^{\circ} \mathrm{C}$ and $-100^{\circ} \mathrm{C}$ curves. Figure 3 shows the atmospheric, lens, and CCD transmission profiles (dotted lines) versus wavelength, and the resulting total SuperWASP transmission (solid line). The transmission profile peaks in the $V$ and $R$ bands (at $\sim 60 \%$ ). Blueward of $V$, both atmospheric extinction and the CCD quantum efficiency reduce throughput. Redward of $R$, the transmission is predominantly reduced by CCD quantum efficiency. We estimated a system zero point using a spectrum of Vega (from Hayes 1985 and Mountain et al. 1985), which we take to represent a zero-magnitude source. Accounting for the collecting area (96.7 $\mathrm{cm}^{2}$ for each lens) and the SuperWASP transmission, we integrated the detected Vega photons across the SuperWASP band. The predicted zero point was found to be $21 \mathrm{mag}\left(2.5 \times 10^{8}\right.$ detected counts $\mathrm{s}^{-1}$ from a zero-magnitude source). As a guide, this means that a signal-to-noise ratio $(\mathrm{S} / \mathrm{N})$ of $\sim 50$ would result from a $30 \mathrm{~s}$ integration on a 16 th magnitude source.

\subsection{Unfiltered Color Term}

We used the spectra of Pickles (1985; available from CDS) and Leggett et al. (2000), ${ }^{2}$ combined with a Johnson $V$ band transmission profile (Bessell 1990) and the SuperWASP passband, to derive synthetic $V$-band and SuperWASP-band flux ratios for early $\mathrm{G}$ to mid $\mathrm{M}$ dwarfs. These flux ratios were converted into SuperWASP colors $\left(V-m_{\mathrm{SW}}\right.$, where $m_{\mathrm{SW}}$ is a

\footnotetext{
${ }^{2}$ From http://www.jach.hawaii.edu/ skl.
} 
magnitude on the natural SuperWASP system) using our Vega spectrum to define zero color. Figure 4 shows the resulting color terms as a function of spectral type. It can be seen that for early G dwarfs the color term is small, but for later spectral types it becomes significant, because most of the optical flux from these dwarfs is not in the $V$ band but is in the red part of the SuperWASP band.

\subsection{Unfiltered Sky Brightness}

To estimate the La Palma sky brightness as seen by SuperWASP, we used the full range of optical sky brightness measurements from Benn \& Ellison (1998) for bright, gray, and dark sky conditions. For each sky, we converted the magnitudes into fluxes using our Vega spectrum (see $\S 3.1$ ). The resulting flux levels were then joined together to provide an approximate sky spectrum. These sky spectra were then multiplied through by the SuperWASP bandpass and converted into SuperWASP magnitudes using our system zero point. The SuperWASP sky brightness estimates were found to be 18.3, 20.3, and $21.1 \mathrm{mag} \operatorname{arcsec}^{-2}$ for bright, gray, and dark skies, respectively. If one accounts for the large SuperWASP pixels and a spatial resolution element containing 12 pixels (a 2 pixel radius aperture), one would expect sky brightnesses of 9.8, 11.8 , and 12.6 mag per resolution element for bright, gray, and dark skies, respectively.

\subsection{Source Crowding with Large Pixels}

The large SuperWASP pixels (and consequent large aperture sizes used for photometry) mean that image crowding will be much more of an issue than it is for higher spatial resolution imaging, particularly in the Galactic plane. This is important for two reasons. First, if a transit source shares its aperture with another star (i.e., if it is blended), the $\mathrm{S} / \mathrm{N}$ of the transit detection will be decreased. However, more importantly, if the point-spread function (PSF) of a neighboring source spills into the aperture of a star, this fraction of the measured flux could vary over time if the PSF changes slightly, or if the chosen aperture does not remain perfectly centered on the starSuperWASP tracking ( $0^{\prime \prime} 01 \mathrm{~s} \mathrm{~s}^{-1}$ accuracy) will cause stars to drift between pixels over several hours. Since we are interested in identifying eclipse dips that are $\sim 4 \%$ deep, we would not want such flux pollution much above the $1 \%$ level. We have therefore used synthetic Gaussian PSFs to determined the distance at which a source (with FWHM $=1.5$ pixels) will pollute its neighbor's 2 pixel aperture by $1 \%$, and we established how this distance $\left(d_{1 \%}\right)$ depends on the relative brightness of the two sources $(\Delta \mathrm{mag})$. We found that

$$
d_{1 \%}=\mathrm{FWHM} \times(1.95-0.12 \Delta \mathrm{mag}),
$$

for $\Delta \mathrm{mag}<2.0$. For fainter neighbors, $d_{1 \%}$ drops more rapidly, reaching zero for $\Delta \mathrm{mag}=5$ (i.e., a factor of 100). So if a

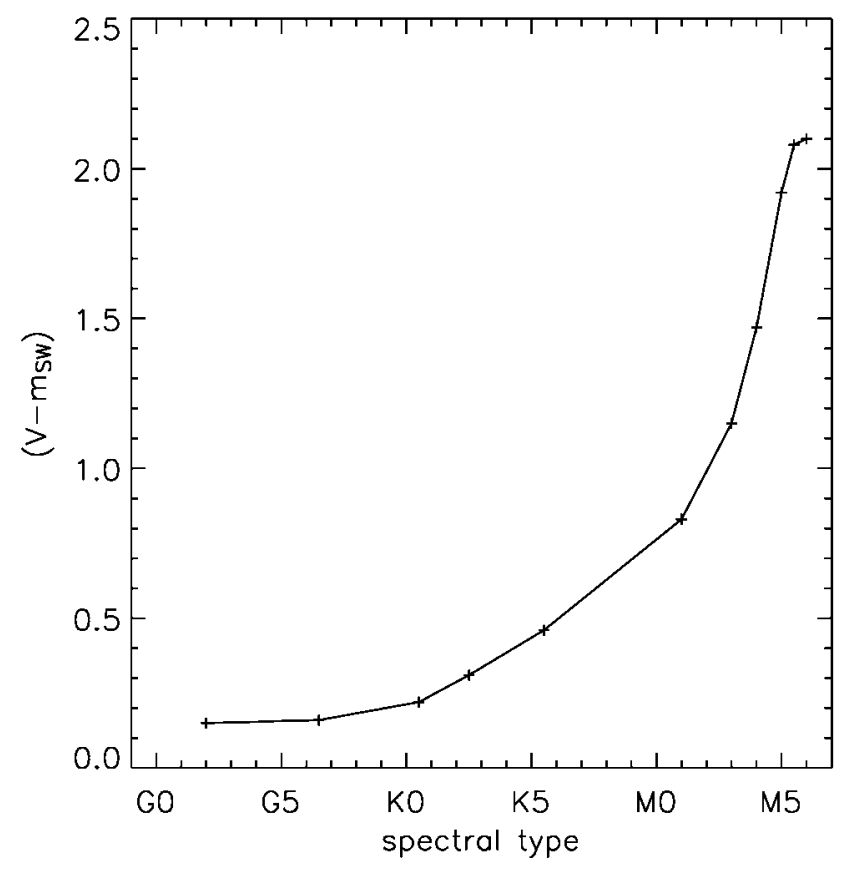

FIG. 4.-Synthetic $V-m_{\mathrm{sw}}$ color vs. dwarf spectral type.

source has a neighbor with $d<d_{1 \%}$, we consider it significantly blended and not measured by SuperWASP with sufficient accuracy for our purposes. We applied this criteria to a series of $1 \mathrm{deg}^{2}$ catalogs (centered on different Galactic coordinates) from the SuperCOSMOS Sky Survey database. ${ }^{3}$ Since we are interested in late $\mathrm{K}$ and early $\mathrm{M}$ dwarfs, we only considered SuperCOSMOS sources with $B_{J}-R \geq 1.8$ and estimated the fraction of blends as a function of Galactic coordinate and source brightness. We found that for $V \sim 16, \sim 30 \%$ of sources with $|b|>20^{\circ}$, and $\sim 75 \%$ of sources with $|b|<20^{\circ}$, will be blended in SuperWASP images. For $V \sim 18$, we found $50 \%$ and $85 \%$ blended fractions for $|b|>20^{\circ}$ and $|b|<20^{\circ}$, respectively.

\subsection{Transit Detection Magnitude Limits}

The $\mathrm{S} / \mathrm{N}$ level of a transit detection is

$$
\mathrm{S} / \mathrm{N}=\left(\frac{R_{\mathrm{BD}}^{2}}{R_{*}}\right)\left(\frac{S_{\mathrm{obs}}}{\text { noise }_{\mathrm{obs}}}\right) \sqrt{\frac{P R_{*}}{t_{\mathrm{obs}} \pi a}},
$$

where $R_{\mathrm{BD}}$ and $R_{*}$ are the radii of the companion $\mathrm{BD}$ and the primary star, $S_{\text {obs }}$ and noise obs are the signals detected from the source and the associated noise during an observation, $P$ is orbital period, $t_{\mathrm{obs}}$ is the time taken for an observation $(\sim 30 \mathrm{~s})$, and $a$ is the separation of the binary. The first term represents the relative decrease in brightness during transit, the second

\footnotetext{
${ }^{3}$ See http://www-wfau.roe.ac.uk/sss.
} 
term is the $\mathrm{S} / \mathrm{N}$ associated with each observation, and the last term is the square root of the number of observations made during a transit. The noise model we used was

$$
\text { noise }_{\mathrm{obs}}=\sqrt{S_{\mathrm{obs}} t_{\mathrm{obs}}+S_{\mathrm{sky}} t_{\mathrm{obs}}+D t_{\mathrm{obs}}+N R^{2}+\mathrm{syst}^{2}}
$$

where $S_{\text {sky }}$ is the signal detected from the sky in an $N=12$ ( 2 pixel radius) spatial resolution element (i.e., the lenses produce a $\sim 1.5$ pixel PSF) during an observation, $D$ is the dark current $\left(\sim 0.01 e^{-} \mathrm{s}^{-1}\right), R$ is the read noise $\left(\sim 12 e^{-}\right)$, and "syst" represents any systematic errors. In general, systematic uncertainties associated with relative photometry of bright sources can be reduced to the 1-2 mmag level (Kane et al. 2004; Bakos et al. 2004). For the fainter sources of interest here, systematics will be dominated by field crowding effects, which sets the upper level of the systematic uncertainties at a self-imposed 10 mmag (see § 3.4).

We define our transit detection limits by requiring a $\mathrm{S} / \mathrm{N} \geq 5$. We would only expect one in $\sim 2$ million stars to register a $5 \sigma$ transit from random noise alone, and since SuperWASP will survey rather less than this number of late K and $\mathrm{M}$ dwarfs, false positives from random noise will not be a problem. We combined equations (2) and (3) with the SuperWASP zero point and used the binary characteristics and stellar model data described in $\S 2.2$ to derive SuperWASP magnitude limits for $5 \sigma$ transit detection. These limits for bright, gray, and dark skies are shown in Figure 5 (solid lines) as a function of primary $M_{V}$. Primary spectral types are also indicated at their appropriate position (taken from Pickles 1985). $V$-band magnitude limits were then derived using the color term from $\S 3.2$ (dashed lines). For comparison, the $\mathrm{G}$ star $10 \sigma$ bright-sky detection limit of Horne (2003) is overplotted (open circle) and joined to its $5 \sigma$ equivalent (filled circle). It is clear from the figure that as one moves to later type stars, transits can be detected out to fainter magnitudes. This is because later stars are smaller, and eclipse dips are correspondingly deeper. In addition, the effect of the broad, unfiltered passband is clear, since SuperWASP will do better for red sources than a comparable $V$-band filtered system.

\subsection{Distance Limits for Transit Detections}

The $V$-band transit detection limits from Figure 5 were converted directly into distance limits using the appropriate values of $M_{V}$. These distance limits are shown in Figure 6 (solid lines) for bright, gray, and dark skies. It can be seen that transits of late $\mathrm{K}$ to early $\mathrm{M}$ dwarfs should be detectable out to $\sim 200$ $500 \mathrm{pc}$. For the later mid M dwarfs, SuperWASP is limited to $\sim 100 \mathrm{pc}$, because of their fainter intrinsic brightness. The figure also contains some additional limits, which are described in the next section.

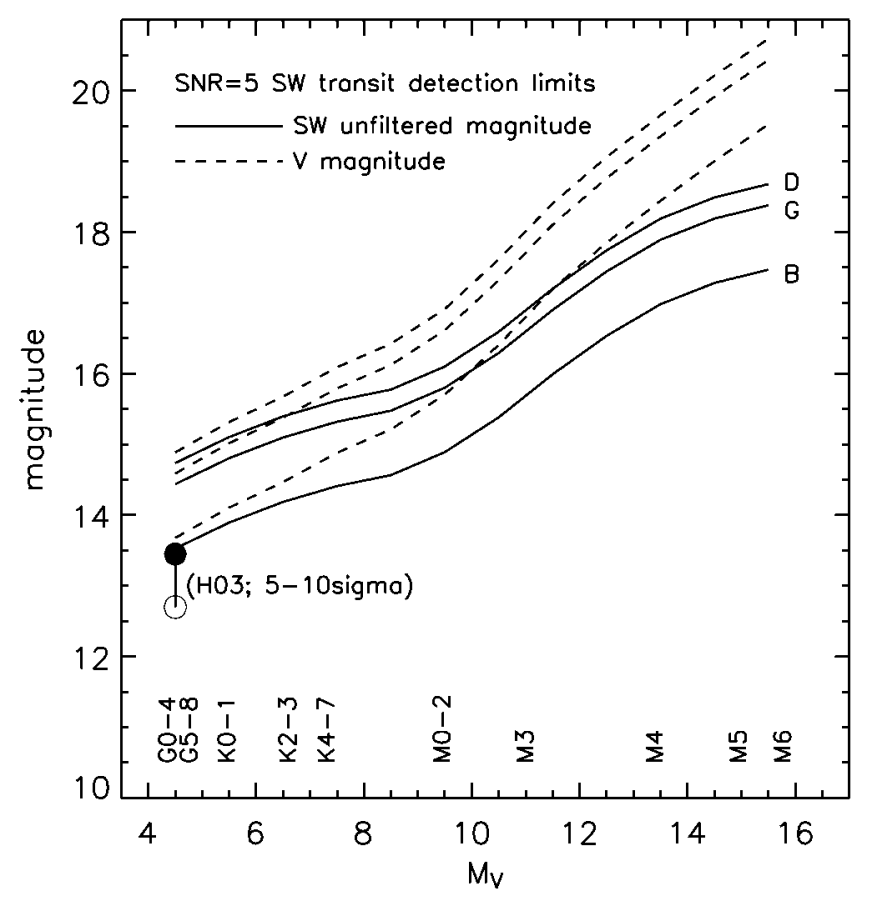

FIG. 5.-SuperWASP magnitude limits for $5 \sigma \mathrm{BD}\left(0.1 R_{\odot}\right)$ transit detections vs. primary type. Limits are shown for dark (D), gray (G), and bright (B) skies. Solid lines show magnitudes in the SuperWASP system. Dashed lines signify the SuperWASP limits converted into $V$ magnitudes. A point from Horne (2003) is shown for comparison.

\section{LIMITS IMPOSED BY FOLLOW-UP REQUIREMENTS}

\subsection{Measuring BD Brightness from Secondary Eclipses}

Having identified a good transit signature of a low-luminosity, fully eclipsing companion (and confirmed it with an intermediate-sized optical telescope), one can then attempt to measure the brightness of the companion from the secondary eclipse. Our ability to do this is governed by the accuracy with which we can measure follow-up light curves and the relative brightness of the companion and primary. Compared to late $\mathrm{K}$ or early M dwarfs, BDs with spectral types below T2 are relatively brightest in the $K$ band (see Leggett et al. 2002). The $K$ band is thus generally the best band in which to search for a secondary eclipse. With $M_{V}=8-12$ and $M_{K}=5-7$ (see Reid \& Cruz 2002), a typical late $K$ or early $M$ dwarf near the SuperWASP distance limits (500-200 pc, respectively) would have $K \simeq 13.5$. The accuracy of $K$-band light curves will depend on photon noise, stability of the instrumental response, and atmospheric conditions. Using 2-4 m class telescopes, a signal-to-photon-noise ratio of $\sim 1000$ could be achievable for a $K \sim 13.5$ source in $\sim 5-10$ minutes. Variations in the zero point and extinction can be corrected for using frequent standard measurements, flat-fielding should be good to at least $0.1 \%$ accuracy, and atmospheric scintillation noise for such obser- 


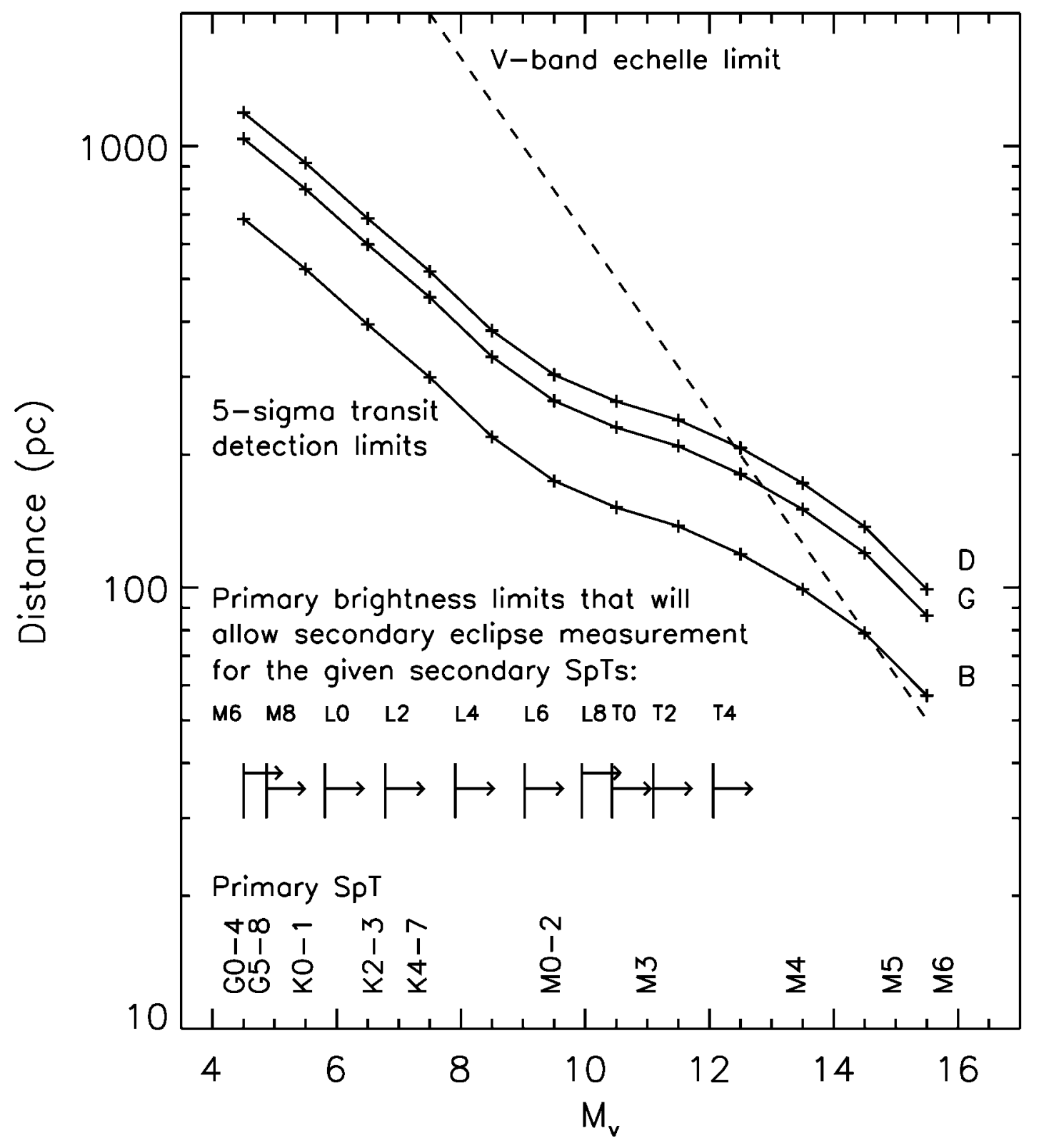

FIG. 6.-Detection and follow-up limits for transiting BD companions. The solid lines show the distance limits for $5 \sigma$ BD transit detections (for dark, gray, and bright skies) vs. primary type. The dashed lines indicate distances out to which the mass of a $0.05 M_{\odot}$ BD at 0.02 AU separation could be accurately measured with $V$ - and $I$-band echelle spectroscopy on an $8 \mathrm{~m}$ telescope. The $M_{V}$ lower limits shown indicate the maximum brightness a primary star may have if we were able to measure the brightness of a BD companion (with spectral type of T4-M6) with 10\% accuracy from a NIR light curve of the secondary eclipse.

vations will be $\sim 1$ part in $10^{4}$ (eq. [10] of Dravins et al. 1998). The main source of systematic uncertainty in near-infrared (NIR) photometry is the amount of atmospheric $\mathrm{H}_{2} \mathrm{O}$ absorption, which can be variable on very short timescales. Kidger \& Martín-Luis (2003) measured high-precision NIR photometry of bright $(K \leq 10)$ northern hemisphere stars using a photometer and were able to obtain $K$-band accuracies (inferred from multiple measurements) as good as $0.4 \mathrm{mmag}$ for some sources (see their Fig. 8). Typical uncertainties would not always be this good, as atmospheric conditions change (e.g., Kidger \& Martín-Luis's median uncertainty was $\sim 3 \mathrm{mmag}$ ). However, an overall $K$-band measurement accuracy of $1 \mathrm{mmag}$ should be a realistic level to aim for. Note that large-scale NIR arrays (such as WFCAM on the UK Infrared Telescope) would allow comparison stars to be measured at the same time as targets, which could reduce atmospheric systematics. However, 
we will continue to use Kidger \& Martín-Luis as our main example, since they have observationally established these levels of accuracy.

The total accuracy with which we can measure the depth of a secondary eclipse dip in a NIR light curve $\left(\sigma_{\text {tot }}\right)$ will depend on both the accuracy of an individual observation $\left(\sigma_{\text {obs }}\right)$ and on the number of data points $(N)$ measured during secondary eclipse (where $\sigma_{\text {tot }}=\sigma_{\text {obs }} \sqrt{N}$; see Henry et al. 2000a). For the binaries we are considering, we would expect an eclipse to last $\sim 1.5 \mathrm{hr}$, and we could thus expect to obtain 6-10 $\mathrm{K}$-band measurements per eclipse (allowing for 5 minutes overhead per observation). By measuring three to four eclipses, and phasing the data appropriately, one could then measure the depth of the secondary eclipse dip with an accuracy of 0.2 mmag. Thus, for an eclipse dip to be measured with $10 \%$ accuracy, it must be at least 2 mmag deep. The BD must therefore be no less than 500 times $(6.75 \mathrm{mag})$ fainter than the primary star. Estimating primary $M_{K}$ from Reid \& Cruz (2002) and BD $M_{K}$ (appropriate for M6-T4 spectral types) from Dahn et al. (2002) and Knapp et al. (2004), we have derived $M_{V}$ brightness limits for primaries with $M_{K} 6.75$ mag brighter than BD companions of each spectral type. These primary $M_{V}$ limits are shown in Figure 6, where each is labeled with its appropriate BD spectral type. As an example, it should be possible to measure the secondary eclipse of an L6 eclipsing BD companion of an $M_{V} \geq 9$ primary.

\subsection{Measuring BD Masses from Radial Velocities}

If a low-luminosity eclipsing companion is detectable from its secondary eclipse, then radial velocity techniques can be employed to accurately measure its mass. For eclipsing systems, the inclination is known to better than $1^{\circ}$, and the relative mass of the $\mathrm{BD}$ (compared to that of the primary) can be determined by measuring a radial velocity curve for the primary. Primary masses will thus act as anchors for the BD masses, and uncertainties in the mass-luminosity relation for low-mass stars (e.g., Henry \& McCarthy 1993) will propagate through to the BD masses. However, it is expected that the low-mass star mass-luminosity relation should improve significantly in the next few years as the orbits of many more $M$ dwarf systems are measured (e.g., by RECONS; Jao et al. 2003).

The radial velocity accuracy required to determine BD masses places its own limits on the distance of useful binary systems. To illustrate this, a $0.05 M_{\odot}$ brown dwarf at $0.02 \mathrm{AU}$ will cause a radial velocity oscillation of $\pm 20 \mathrm{~km} \mathrm{~s}^{-1}$ for a $0.5 M_{\odot} \mathrm{M}$ dwarf primary. Standard arc lamp echelle spectroscopy is limited by several factors: zero-point drifts in the wavelength scale between exposures, the spectral features available for cross-correlation techniques, the presence of telluric absorption, the $\mathrm{S} / \mathrm{N}$ of the spectra themselves, and intrinsic properties of the star (surface spots or rotation). We return to the issue of spots and rotation in $\S 7.3$. For the other factors, however, we use previous optical studies with $8 \mathrm{~m}$ telescopes as a guide. Reid \& Mahoney (2000) observed zero-point drifts of $\pm 1 \mathrm{~km} \mathrm{~s}^{-1}$ during a full night for the Keck HIRES echelle spectrograph. However, they managed to obtain $\pm 300 \mathrm{~m} \mathrm{~s}^{-1}$ accuracy by cross-correlating echelle orders (containing strong telluric signatures) with white dwarf spectra (which act essentially as continuum sources onto which a clear telluric signature is imprinted). More recently, Konacki et al. (2003) used an iodine cell to more rapidly measure zero-point drifts of $\sim 200 \mathrm{~m} \mathrm{~s}^{-1}$ over timescales of $\sim 30$ minutes. These studies looked mostly at $\mathrm{K}$ and $\mathrm{M}$ dwarfs from 3850 to $6200 \AA$ and 6350 to $8730 \AA$, respectively, and found that such drifts generally dominate the overall uncertainty, with contributions of only $\pm 60 \mathrm{~m} \mathrm{~s}^{-1}$ coming from the $\mathrm{S} / \mathrm{N}(\sim 15-25)$ of the spectra themselves.

It is thus clear that optical echelle spectroscopy should be capable of providing radial velocities with accuracies of \pm 0.5 $\mathrm{km} \mathrm{s}^{-1}$ (enough to constrain close BD companion masses at the $\sim 2 \%-3 \%$ level) using lower $\mathrm{S} / \mathrm{N}$ spectra from rather fainter sources with $V \sim 19$. We have converted this magnitude limit into a distance limit, which is shown in Figure 6 (dashed line). It can be seen that for sources with spectral types below M4, the echelle follow-up requirements do not impose any additional distance constraints beyond those of SuperWASP itself. The efficient follow-up of later type primaries at the SuperWASP distance limit would require a NIR echelle spectrograph (such as Phoenix on Gemini). However, we do not expect to find many of these to be fully eclipsing systems (see $\S \S 2.2$ and 6).

\section{DETERMINING ACCURATE AGES}

\subsection{Open Clusters}

Disk stars with the best constrained ages are those in open clusters. The age of an open cluster can be well constrained using a variety of methods, such as fitting the upper main sequence turnoff (Sarajedini et al. 1999) or, for young clusters, measuring the magnitude of the lithium depletion edge (Stauffer et al. 1998). An open cluster is believed to be a coeval association (with only a relatively small age spread of perhaps a few Myr). Therefore, if membership of a cluster is established, then the cluster age can be assumed for the star. However, the majority of disk stars are not open cluster members. Within the SuperWASP distance limits $(\sim 500 \mathrm{pc})$, there are $\sim 31$ open clusters in the La Palma sky (see the LEDAS database ${ }^{4}$ ). Although cataloged membership of these clusters is by no means complete, a reasonable estimate would be that between them they contain no more than a few thousand late $\mathrm{K}$ or early $\mathrm{M}$ dwarfs. This represents a tiny fraction of the number of noncluster disk stars of this type in the SuperWASP sky ( 1 million). Open cluster membership will thus not be very useful

\footnotetext{
${ }^{4}$ At http://ledas-www.star.le.ac.uk
} 
TABLE 2

Summary of the Kinematic Moving Groups that Make Up the Local Young Disk Population

\begin{tabular}{|c|c|c|c|c|c|}
\hline Moving Group & $\begin{array}{l}U, V, W \\
\left(\mathrm{~km} \mathrm{~s}^{-1}\right)\end{array}$ & $\begin{array}{l}\text { Age } \\
(\mathrm{Myr})\end{array}$ & Includes & Comments & References \\
\hline IC $2391 \ldots \ldots \ldots \ldots$ & $-21,-16,-9$ & $35-55$ & IC 2391 cluster & & 1 \\
\hline Gould Belt ........... & $U \sim V \sim W \sim-20$ to 0 & $10-90$ & $\begin{array}{l}\text { Sco-Cen, Cas-Tau, Carina-Vela, } \\
\text { and TW Hydrae associations }\end{array}$ & $\begin{array}{l}\text { Velocity ellipsoid with some substructure } \\
\text { (some structures sometimes associated } \\
\text { with the Pleiades MG). }\end{array}$ & $2,3,4$ \\
\hline \multirow[t]{2}{*}{ Pleiades .............. } & $-9,-26,-9$ & $100-150$ & Pleiades, $\alpha$ Per, and NGC 2516 clusters & $\begin{array}{l}\text { Aka the Local Association; contains two } \\
\text { main velocity substructures. }\end{array}$ & 5,6 \\
\hline & $-17,-22,-6$ & $200-300$ & M34 cluster & & \\
\hline Castor ................. & $-11,-8,-10$ & $\sim 320$ & A stars Vega and Fomalhault & & 7 \\
\hline Coma Berenices ...... & $-10,-5,-8$ & $250-400$ & Coma Ber cluster (aka Melotte 111) & & 8 \\
\hline Ursa Major ........... & $+15,+1,-11$ & $400-600$ & Ursa Major cluster & Aka the Sirius supercluster. & 9 \\
\hline NGC $1901 \ldots \ldots \ldots . .$. & $-25,-10,-15$ & $\sim 500$ & NGC 1901 cluster & & 10 \\
\hline Hyades............ & $-40,-18,-2$ & $\sim 600$ & Hyades and Praesepe clusters & & 11 \\
\hline
\end{tabular}

References. -(1) Lyngå 1987; (2) Moreno et al. 1999; (3) Makarov \& Urban 2000; (4) Song et al. 2003; (5) Asiain et al. 1999a; (6) Asiain et al. 1999b; (7) Ribas 2003; (8) Odenkirchen et al. 1998; (9) King et al. 2003; (10) Dehnen 1998; (11) Montes et al. 2001.

for constraining the age of a significant fraction of the binary systems under consideration.

\subsection{Young Moving Groups}

Open clusters are thought to represent only small components of larger kinematically distinct coeval populations that are spatially dispersed within the disk. Such disk populations are known as moving groups and were originally identified by Olin Eggen in the 1950s. However, it has since become clear that many of the older ( $>1$ Gyr) so-called moving groups he identified show a spread in both age and metallicity (Nordström et al. 2004), and these kinematic signatures are more likely the result of disk heating by stochastic spiral waves (e.g., De Simone et al. 2004). There is evidence, however, that the younger moving groups are coeval. Castro et al. (1999) found identical $\mathrm{Cu}$ and $\mathrm{Ba}$ abundances for seven $\mathrm{G}$ and $\mathrm{K}$ dwarfs in the Ursa Major moving group, and King et al. (2003) found clear evidence of a correlation between membership of the Ursa Major moving group and activity levels (from $\mathrm{Ca}$ II $\mathrm{H}$ and $\mathrm{K}$ emission strength).

Such young moving groups are thought to originate in the same environments as open clusters. Very young clusters should virialize somewhat within their natal gas, and when the gas is cleared by OB star action and the cluster expands, the stars with high enough velocities will become unbound. These unbound stars then slowly expand, forming the moving group and possibly leaving behind an open cluster consisting of any remaining bound stars (see the $N$-body simulations of Kroupa et al. 2001). Typical expansion velocities for the unbound population will be $\sim 5-10 \mathrm{~km} \mathrm{~s}^{-1}\left(5-10 \mathrm{pc} \mathrm{Myr}^{-1}\right)$, and the moving group's space motion will thus remain quite well defined (superimposed on the more dispersed disk population) until it has had time to become significantly phase-mixed by disk heating. Models suggest that phase mixing will increase a moving group's velocity dispersion by $\sim 20 \mathrm{~km} \mathrm{~s}^{-1}$ over $\sim 1$ Gyr (Asiain et al. 1999a). Moving groups younger than $\sim 1$ Gyr should thus consist of young populations with characteristic space motions, and membership of such a group will accurately constrain the age of an eclipsing BD-M dwarf binary just as it would do for open cluster members.

The most notable such groups in the solar neighborhood are listed in Table 2, along with their kinematic motions and age. Most have open cluster(s) associated with them, which range in size from the rich Pleiades cluster down to the almost evaporated Ursa Major cluster. In general, each group has one (or two, in the case of the Pleiades) distinct kinematic signature. However, note that the Gould belt is rather more complex than the others, since it consists of an agglomeration of several very young kinematic groups superposed on a less distinct velocity ellipsoid (Moreno et al. 1999). Each of the distinct kinematic subgroups is believed to have had a common origin (e.g., Asiain et al. 1999b), with the ellipsoid possibly resulting from an expanding ring of star formation (the Local Bubble). Interestingly, it appears that a large fraction of young local stars reside in these moving groups. Dehnen (1998) has shown (using Hipparcos astrometry) that the majority of color-selected young disk stars are moving-group members, and Makarov (2003) found that a high fraction of X-ray active stars within $50 \mathrm{pc}$ belong to the Pleiades and Gould Belt moving groups.

\subsection{Identifying Young Potential Moving-Group Members}

In order to separate possible young moving-group members from the large population of older disk stars, one needs to observationally identify young sources. Age constraints can be placed on young late $\mathrm{K}$ and early $\mathrm{M}$ dwarfs in a variety of ways. Chromospheric or coronal $\mathrm{H} \alpha$ emission from open cluster members (Hawley et al. 1999; Gizis et al. 2002) has shown that there is a well-defined, age-dependent color beyond which activity becomes ubiquitous. Despite considerable scatter, all stars redder than this color are dMe's $\left(\mathrm{EW}_{\mathrm{H} \alpha} \geq 1.0 \AA\right)$, while 
the bluer stars are dM's without emission. This so-called $\mathrm{H}_{\alpha}$ limit color increases with the age of the population and can identify (in emission) K5-M0 dwarfs younger than $~ 30 \mathrm{Myr}$, M0-M3 dwarfs younger than 100 Myr, and M3-M5 dwarfs younger than $\sim 600 \mathrm{Myr}$. Lithium abundances can also provide age constraints. Late $\mathrm{K}$ dwarfs deplete lithium over $\sim 100 \mathrm{Myr}$, and early-to-mid $\mathrm{M}$ dwarfs deplete lithium over $\sim 30-50 \mathrm{Myr}$ (Preibisch et al. 2001; Zapatero Osorio et al. 2002; Jeffries et al. 2003; Randich et al. 2000). One can also constrain ages by fitting isochrones to color-magnitude data. A $\sim 0.5 M_{\odot}$ star will not have fully contracted until it is $\sim 100 \mathrm{Myr}$ old. Therefore, with an accurate parallax distance (possible even at $500 \mathrm{pc}$, using the VLT as an interferometer), an appropriate CMD (see Stauffer et al. 2003), and photometric corrections to account for metallicity-sensitive colors (e.g., Kotoneva et al. 2002), the age of such a star could be approximately constrained.

Clearly, identifying late $\mathrm{K}$ and early $\mathrm{M}$ dwarfs younger than $\sim 100$ Myr will not be difficult. However, older counterparts will be fully contracted, show no lithium, and have $\mathrm{H}_{\alpha}$ emission below $1 \AA$. Such sources will require higher resolution analysis if their ages are to be constrained from their activity levels. For instance, one could gauge activity levels by measuring $\mathrm{Mg}$ II $\mathrm{H}$ and $\mathrm{K}$ and Ca II H and K (Doyle et al. 1994; Christian et al. 2001; Mathioudakis \& Doyle 1991) or by measuring $\mathrm{H} \alpha$ at high resolution, and look for weaker line emission in the core of the absorption line (e.g., Short \& Doyle 1998). These methods allow one to study activity levels down to those associated with old disk M dwarfs (see Doyle et al. 1994) and should therefore provide an effective way to separate young $(<1 \mathrm{Gyr})$ late $\mathrm{K}$ and early $\mathrm{M}$ dwarfs from older disk stars.

\subsection{Kinematic Membership of Moving Groups}

Establishing kinematic membership of moving groups will require space motions accurate to a few kilometers per second. Since accurate radial velocity curves will have been previously measured (to determine BD mass; see $\S 4.2$ ), these will provide very accurate center-of-mass radial velocities for the binaries. Although it should be possible to derive some proper motions (with the required accuracy) from existing archive measurements (e.g., with SuperCOSMOS), sources with distances near $500 \mathrm{pc}$ would require some additional effort. Proper motion accuracies would need to be at the milliarcseconds per year

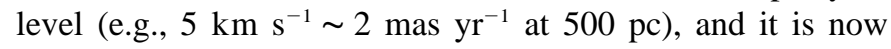
quite feasible to measure proper motions of this accuracy using adaptive optics technology with a baseline of $\sim 1-2$ yr (cf. 3 mas positional accuracy; Close et al. 2003b).

The confidence with which one can assign kinematic membership in a particular moving group will depend on how unique the group's measured kinematic signature is compared to other young moving groups and any non-moving-group population of young stars (see Figs. 1 and 2 of Montes et al. 2001). The Hyades moving group, for instance, has a space motion well separated from the local standard of rest but has a rather larger internal dispersion compared with some younger moving groups. As a result, some Hyades moving-group members will not be obvious kinematic members of the group. At the other extreme, the very young members of the Gould belt and the Pleiades moving-group components will not show as much internal dispersion (within each component). However, because of their similar space motions, there is some kinematic overlap between some of these components. Furthermore, young stars that are not members of any moving group can act as a source of potential contamination if they have the same space motion as the group. However, if the majority of young stars are moving-group members (see $§ 5.2$ ), such contamination should not be at a high level and will only decrease membership probabilities by a small amount.

While it is clear that only some fraction of moving-group members will be easily identifiable from their kinematics, it is by no means clear exactly what this fraction will be, since there are insufficient radial velocity measurements of young disk stars to properly characterize our expectations. However, it is worth noting that moving-group characterization will certainly improve in the near future as large-scale radial velocity surveys (such as RaVE; Steinmetz 2003) provide radial velocity and metallicity for complete magnitude-limited samples.

\section{SIMULATING THE NUMBER OF USEFUL ECLIPSING BD COMPANIONS}

We describe here a simulation we have made to predict the size and properties of the population of fully eclipsing BD companions that could be identified by the SuperWASP survey over the whole sky. This simulation predicts how many BDs will be identified, how many of these will be detectable from their secondary eclipse, how many are likely to be significantly irradiated by their primary, and how many will be young (and thus potentially moving-group members).

\subsection{The Simulation}

For our simulation, we consider low-mass stars with BD companions in close $(\sim 0.02 \mathrm{AU})$ orbits. These binaries will have short orbital periods of 1-1.5 days. We thus expect $\sim 40-60$ transits per binary during a $\sim 2$ month SuperWASP monitoring period, $\sim 11-16$ of which should occur while SuperWASP is taking data (allowing for typical $9 \mathrm{hr}$ nights and $\sim 25 \%$ time lost to bad weather). SuperWASP should experience $\sim 9.5$ dark nights per lunation, and the Moon will be set for typically 20\% of nondark nights. We therefore expect dark sky conditions $\sim 45 \%$ of the time and would expect approximately five to seven transits to occur during this time. It is therefore appropriate to use the dark-sky distance limits for transit detection. We also assumed that for late $\mathrm{K}$ dwarfs $(V \sim 16$ near the SuperWASP distance limits), $30 \%$ and $75 \%$ will be lost due to blending for $b>20^{\circ}$ and $b<20^{\circ}$, respectively. For the early $\mathrm{M}$ dwarfs $(V \sim 18), \sim 50 \%$ and $85 \%$ will be blended for $b>20^{\circ}$ and $b<20^{\circ}$, respectively (see $\S 3.4$ ). We therefore chose to consider 


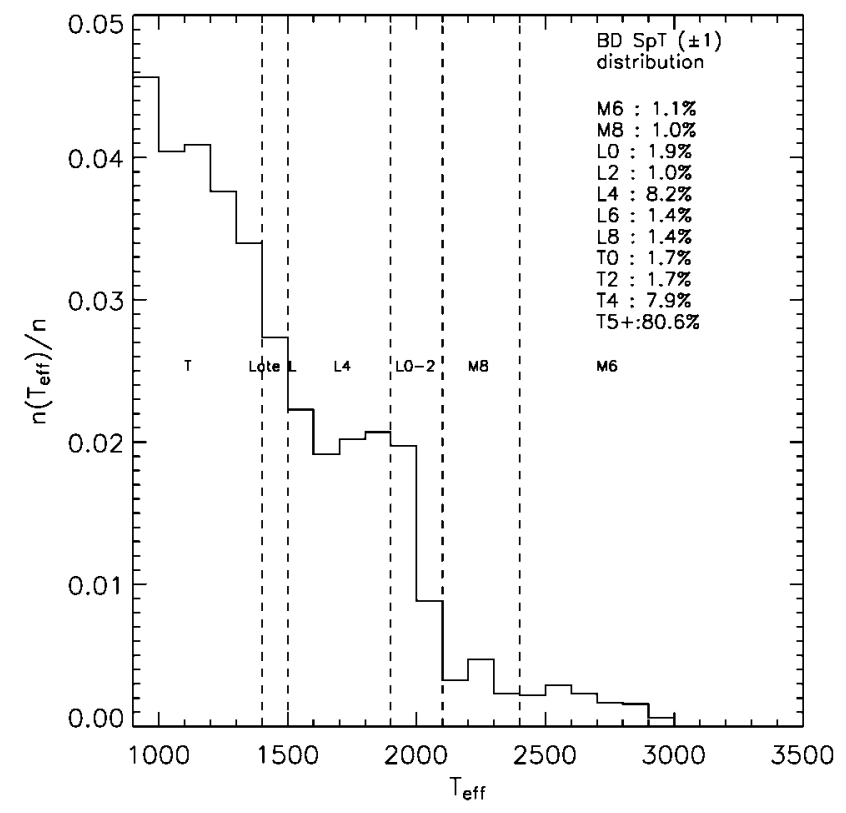

FIG. 7. $-T_{\text {eff }}$ distribution for a population of BDs with a uniform age spread from 0 to $10 \mathrm{Gyr}$ and an $\alpha=0.5$ mass function. Some spectral type divisions are indicated with dashed lines. The fraction of BDs in spectral type ranges from M6 to T4 is given in the top right section of the plot. Note that many BDs are cooler than $900 \mathrm{~K}$, although the plot does not cover this $T_{\text {eff }}$ range.

only regions of sky with $b>20^{\circ}$ and combined this sky coverage with our distance sensitivities to derive space volumes. We then combine these volumes with the nearby luminosity function of Reid et al. (2002) and the vertical disk density law from Zheng et al. (2001) to derived the number of stars within the transit detection limits as a function of $M_{V}$. We then factored in a $1 \% \mathrm{BD}$ companion fraction at a separation of $0.02 \mathrm{AU}$ for late $\mathrm{K}$ and $\mathrm{M}$ dwarfs $\left(M_{V} \geq 8\right)$, with a negligible binary fraction for earlier stars (see $\S 2.1$ ), and thus determined the fraction of binaries that should be fully eclipsing using the equations from $\S 2.2$.

In order to factor in how many of these BDs will be detectable from the secondary eclipse, we simulated the BD $T_{\text {eff }}$ distribution. This was done by defining an array of $\sim 8000$ BDs $\left(0.015-0.075 M_{\odot}\right)$ in wihch we assumed a population mass function with $\alpha=0.5$ and a uniformly distributed random age distribution from 0 to $10 \mathrm{Gyr}$. For each BD, we then used the NextGen and "dusty" (Chabrier et al. 2000) model isochrones to estimate $T_{\text {eff }}$ from mass and age, using linear interpolation between isochrone grid points. We chose NextGen $T_{\text {eff }}$ 's higher than $2500 \mathrm{~K}$ and dusty $T_{\text {eff }}$ 's for instances in which the NextGen $T_{\text {eff }}$ 's were below $2500 \mathrm{~K}$. The resulting histogram of $T_{\text {eff }}$ ' $s$ is shown in Figure 7. Spectral type ranges (some shown on the figure) were determined using the spectral type- $T_{\text {eff }}$ relations from Reid et al. (1999; M6-L2) and Knapp et al. (2004; for >L2). For each of our selected spectral type ranges (M6 \pm 1 to T4 \pm 1 ), we added up all the BDs in the appropriate $T_{\text {eff }}$ range and divided by the total number, so as to
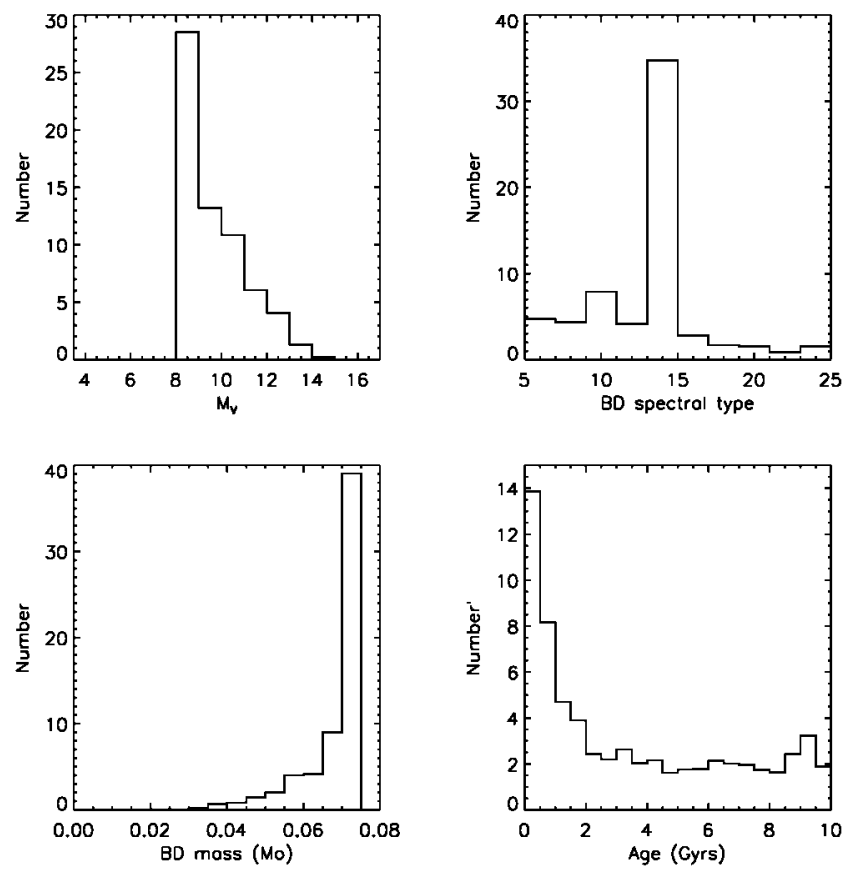

FIG. 8.-Predicted numbers of fully eclipsing brown dwarf companions with measurable secondary eclipses that could be found by SuperWASP. The four panels show the primary and BD spectral type distributions and the BD mass and age distributions.

represent the fraction of BDs in that range. These results are tabulated in Figure 7. The implications of this $T_{\text {eff }}$ distribution are discussed in the next section. Finally, we removed any binaries in which the primary was too bright compared to the BD companion (using the $M_{V}$ limits determined in $\S 4.1$ ).

\subsection{Simulation Results}

The simulation predicts that a total of $\sim 1000$ eclipsing BD companions could be discovered by SuperWASP over the full La Palma sky. Of these, secondary eclipse measurements should allow the BD brightness to be measured (to $10 \%$ accuracy) for $\sim 150$ systems. The predicted distribution of primary and BD spectral types and the BD mass and age for these 150 systems is shown in Figure 8. Note that these predicted results scale with the assumed BD binary fraction and 1/a and can thus be easily scaled up or down for different input values. In the top left histogram, the favored primary spectral types are the earlier types, since these stars are brighter and are detectable out to greater distances. In the top right histogram, there is a strong preference for a BD spectral class of L4 \pm 1 . This preference results from three factors in our simulation. First, the spectral type- $T_{\text {eff }}$ relation we used (Knapp et al. 2004) gives a relatively large $T_{\text {eff }}$ range for the L3-L5 spectral classes (1500-1900 K; see Fig. 7). Secondly, L4 companions will be detectable around all the primaries that our simulation considers as potential hosts for BD companions $\left(M_{V}>8\right.$; see Fig. 6). 
Thirdly, although we assume an approximate BD upper mass limit of $0.075 M_{\odot}$, the dusty models predict a hydrogen-burning minimum mass $m_{\text {нвмм }} \sim 0.07$. Our simulation thus has the higher mass $\left(0.07-0.075 M_{\odot}\right)$ objects stabilizing their $T_{\text {eff }}$ around $1500-1900 \mathrm{~K}$ (i.e., $\mathrm{L} 4 \pm 1$ ) in the $1-10 \mathrm{Gyr}$ age range. Uncertainties in the spectral type- $T_{\text {eff }}$ relation and the precise value of $m_{\text {нвмм }}$ could thus change the spectral type and width of the peak somewhat. However, note that we certainly expect an L4 predominance over later companions, since the L4's are detectable around the brighter primaries. The bottom left histogram shows this preference for $0.070-0.075 M_{\odot}$ objects. Finally, the bottom right histogram shows a strong preference for young BDs, because young BDs are brighter and more easily detectable from their secondary eclipses. The age histogram has $\sim 20$ binaries younger than $1 \mathrm{Gyr}, \sim 10$ with ages 1-2 Gyr, and a continuum of $\sim 2 \mathrm{Gyr}^{-1}$ for 2-10 Gyr. This relatively flat age continuum is mainly populated by the $\mathrm{L} 4 \pm 1$ type companions.

But how many of these detectable BDs would be significantly irradiated? It can be seen by looking at Figure 2 that no BDs with spectral types $\leq \mathrm{L} 0$ would be significantly irradiated by any of the primaries we consider. However, an L4 BD would be slightly $(\leq 25 \%)$ irradiated by the $M_{V}=8-9$ primaries. Later BDs would be irradiated by steadily fainter primaries, and the small number of T5 BDs would be irradiated by the majority of primaries. Using Figure 2 and the top two histograms in Figure 8 , we estimate that $\sim 70 \%$ of the predicted companion BDs will not be irradiated by more than $10 \%$.

\subsection{A Subset of Young Systems}

We also wish to consider the properties of a young subset of the BDs. Such systems are preferred by our detection criteria, because BDs are brighter when young. As we discussed in $\S 5$, many young systems should be members of kinematic moving groups and, as such, have ages that can be well constrained. We therefore reran our simulation using a $T_{\text {eff }}$ distribution derived by only selecting BDs with ages $\leq 1 \mathrm{Gyr}$. This simulation predicted $\sim 20$ binary systems in which BDs could be well measured from the secondary eclipse. The histograms describing these binaries are shown in Figure 9. The primary spectral type histogram is similar in shape to that of the main simulation. The BD spectral type histogram, however, is somewhat different from the main simulation. The L4 peak is still there but has been significantly reduced in number. This is because although the L3-L5 $T_{\text {eff }}$ range is still relatively large, the majority of the $0.070-0.075 M_{\odot}$ companions (i.e., in the 1-10 Gyr age range) have been excluded in this age-limited simulation. However, there is now an additional peak for M5-M9 BDs. This peak results from the fact that all M5-M9 dwarfs must be young in order to be BDs and will thus not be removed by our age constraint. The mass histogram still shows a general preference for higher mass BDs, but the removal of the large number of older $0.070-0.075 M_{\odot}$ objects has decreased the highest mass point significantly. The constrained age histogram is shown
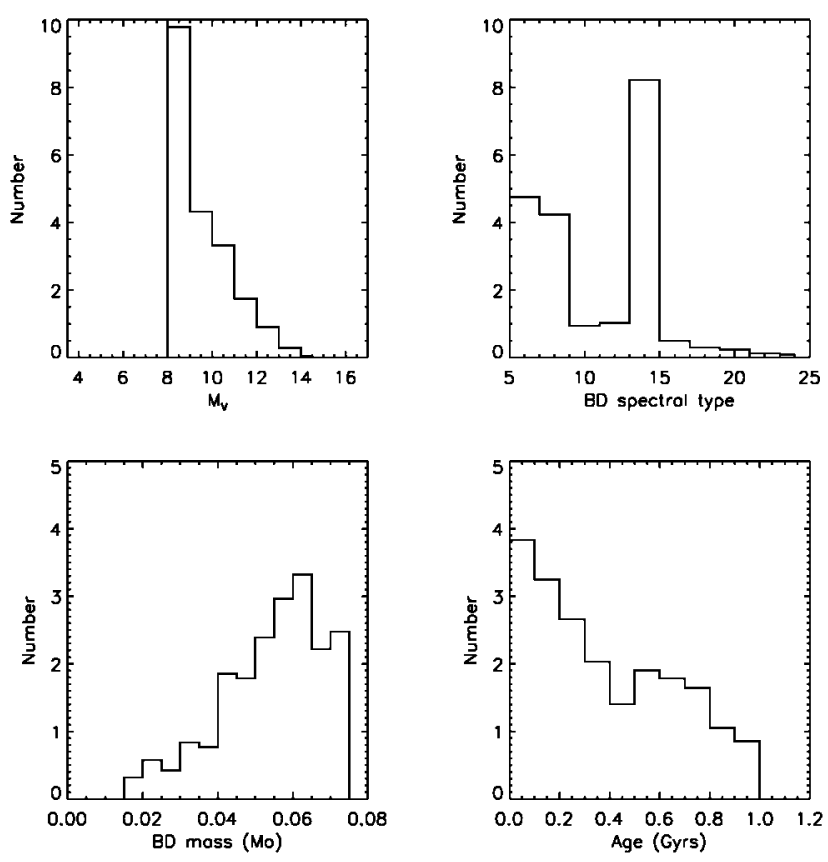

FIG. 9.- Same as Fig. 8, but for systems with ages $<1$ Gyr.

with a finer scale, and the preference for younger systems clearly remains. As before, we have used Figure 2 and the top two histograms (this time from Fig. 9) to estimate irradiation effects, and we estimate that $\sim 80 \%$ of the predicted young BD companions will not be irradiated by more than $10 \%$. The fraction of the $\sim 16$ binaries (containing unirradiated BD companions) that will be clearly associated with a moving group is unclear (see § 5.4). However, it seems likely that we might expect $\sim 10$ systems capable of providing BD mass, age, radius, metallicity, and luminosity data for our assumed $1 \%$ BD companion fraction.

\section{IDENTIFICATION AND FOLLOW-UP}

\subsection{Detection Rate}

Online optical survey data suggest that we would expect $\sim 300,000$ to 1 million sources to $V \sim 18$ in a $320 \mathrm{deg}^{2}$ area of sky with $|b|>20^{\circ}$ (i.e., a typical SuperWASP-I pointing). Typically, $\sim 70 \%$ of these will have $V=16-18$, and $\sim 20 \% V=$ $14-16$. About $20 \%$ of sources will be late $\mathrm{K}$ and early $\mathrm{M}$ dwarfs ( 100,000 per $\left.320 \mathrm{deg}^{2}\right)$, with the majority being earlier $\mathrm{G}$ and $\mathrm{K}$ dwarfs. Of these, $\sim 20,000$ will be monitored with a sufficient accuracy to detect BD transits (i.e., mostly in the $V=$ 14-16 range). Typically, $\sim 30 \%$ ( 6000) of these are expected to be slightly blended (at the $\geq 1 \%$ flux level) with neighboring sources. We do not expect random fluctuations to produce more than $\sim 1$ false detection of a $5 \sigma$ transit event in every $\sim 2$ million sources (i.e., in the full-sky late $\mathrm{K}$ and $\mathrm{M}$ dwarf population). False positives will more likely result from unresolved eclipsing stellar systems in which the eclipsing binary component con- 
tributes only a small fraction of the total light. Alonso et al. (2004) report the discovery of one transiting planet out of 16 candidates (where the false positives were dominated by such sources).

We might expect about five genuine fully eclipsing BD companions to be discovered in each $\sim 320 \mathrm{deg}^{2}$ area of sky (or, for instance, $30 \mathrm{yr}^{-1}$ from SuperWASP-I). Of these, we would expect $\sim 15 \%$ of the binaries $\left(\sim 5 \mathrm{yr}^{-1}\right)$ to have measurable $(10 \%$ accuracy) secondary eclipses, and $\sim 1 / 3$ of these to have ages under 1 Gyr (i.e., 1-2 $\mathrm{yr}^{-1}$ ). These BD detection rates can be scaled for alternative binary criteria - they will scale with BD companion fraction and 1/a. Note also that the actual transit candidate numbers will be higher, since we have only considered full eclipses, and we also expect additional transits by faint low-mass stellar companions and possibly planetary companions to pass our selection criteria. However, the level at which these will occur is beyond the scope of this paper.

\subsection{Follow-up Procedures}

The task of identifying eclipsing BD companions will begin with a search of the transit survey database. Automated search algorithms should be used to identify transit signatures in the light curves, allowing for the possibility that there could also be some variation in brightness due to rotationally modulated spot coverage. Large pixels may result in the blending of some sources, so it will be important to confirm the transit source at better spatial resolution. Optical (e.g., from the STScI Digitized Sky Survey or the SuperCOSMOS Sky Survey) and near-infrared (from the Two Micron All Sky Survey) sky survey images will show if a transit source is a blend of several sources or is a single star and will also provide optical/NIR colors and basic astrometry. These data should also differentiate between dwarfs and giants, confirming or otherwise the faint nature of a transiting companion. It may well also be desirable at this stage to measure additional optical light curve data (using a larger telescope) to both confirm the transit source and more accurately measure the primary eclipse profile. With the addition of a measured spectral type (and metallicity), the character of the transit will have been well established.

Having confirmed a low-luminosity eclipsing companion of a cool dwarf, the next step will be to attempt to measure the secondary eclipse profile and obtain high-resolution radial velocity data (see $\S \S 4.1$ and 4.2). A parallax distance to the primary will also be required to confirm its brightness (from which the BD brightness is inferred). Together, these data could provide BD mass, radius, metallicity, and brightness. The final stage will be to try to determine the age of the system by measuring accurate astrometry and considering all relevant moving-group membership criteria (see $§ 5.3$ ).

\subsection{Additional Issues for Young Sources}

Many young stars may show periodic photometric variability resulting from rotationally modulated spot coverage. This gen- erally occurs at the 5\%-10\% level (Krishnamurthi et al. 1998), has periods of $\sim 1-5$ days (Terndrup et al. 2000), and maintains the same modulation shape over numerous rotation periods (Young et al. 1990). The binary systems we consider should produce primary eclipses at least $4 \%$ deep, lasting for $\sim 1.5 \mathrm{hr}$, with a period of $\sim 1.5$ days. With such distinct forms of variation, automated search algorithms should be able to identify transit sources even if they show some spot modulation.

Accurately measuring the much fainter secondary eclipses from follow-up NIR light curves of variable sources could prove more of a challenge. If the primary rotation and $\mathrm{BD}$ orbital periods are different, one could simply determine the form of the rotational modulation by phasing the data appropriately and averaging over several rotation periods. The rotational modulation could then be subtracted off the light curves, leaving only the secondary eclipse dips. However, if the rotation and orbital periods are the same (close binaries can become phase-locked by tidal forces; see below), then the eclipse times will always correspond to the same rotational phase.

For a $0.05 M_{\odot} \mathrm{BD}$ at $0.02 \mathrm{AU}$ separation from a primary with mass from 0.6 to $0.35 M_{\odot}$ (late $\mathrm{K}$ to early $\mathrm{M}$ ), the spinorbit synchronization timescale varies from $\sim 0.04$ to $2.5 \mathrm{Gyr}$, respectively (depending primarily on the primary radius and companion orbital separation; Udry et al. 2002). Such close $\mathrm{BD}$ companions around late $\mathrm{K}$ dwarfs are thus likely to be in synchronized orbits, but those around early $\mathrm{M}$ dwarfs will probably not have had time to synchronize. To deal with synchronized sources, one could measure simultaneous $R_{-}^{-}, I_{-}^{-}$, and $K-$ band light curves. The optical bands would contain a negligible fraction of BD flux, and any variability would thus be purely due to rotational modulation. The color-color relationship between $R-K$ and $R-I$ modulation away from eclipse could be well determined from the light curve data. This relationship will depend on how the flux in each band changes as spots rotate onto, around, and off the observable face of the star. This will of course depend on the temperature, size, and surface distribution of spots, but there should be a tight relationship for any particular spot configuration. This color-color relationship could thus be used to convert $R-I$ measurements (taken during eclipse) into $R-K$ measurements (as if the BD were not eclipsing). The $R$-band light curve could then be converted into a "pseudo- $K$-band light curve" (using this $R-K$ color), which would show only the $K$-band rotational modulation during eclipse. This could then be subtracted off the original light curve as before. Simultaneous optical/NIR observations would be best done with a robotic telescope with infrared/optical capabilities. The $2 \mathrm{~m}$ robotic Liverpool Telescope, for example, could measure an appropriate set of $R-, I-$, and $K$-band magnitudes in $\sim 25$ minutes. This would then require light curve coverage of more transits to obtain the required accuracy.

The radial velocity data could also be affected by rotationally modulated spot coverage. As photospheric dark spots and brighter regions rotate over the surface of the star, they can 
produce radial velocity modulation. These rotational radial velocity variations will have an amplitude up to $v \sin i$, a period equal to the rotation period $\left(P_{\text {rot }}\right)$, and an oscillatory shape that depends on the distribution of spots across the stellar surface. For illustration, early-M stars with ages under $100 \mathrm{Myr}$ fall into three categories of rotation rate: the majority $(\sim 60 \%-70 \%)$ have slower $v \sin i<20 \mathrm{~km} \mathrm{~s}^{-1}$ rotation velocities (with rotation periods of $\sim 1-5$ days); $\sim 10 \%-20 \%$ are faster rotators ( $v \sin i=30-60 \mathrm{~km} \mathrm{~s}^{-1}$ ); and only 5\%-20\% are ultrafast rotators, with $v \sin i>100 \mathrm{~km} \mathrm{~s}^{-1}$ (see Mochnacki et al. [2002] and Terndrup et al. [2000] for young field stars and Pleiades stars, respectively). The radial velocity oscillation that we expect close BD companions to induce is $\sim \pm 20 \mathrm{~km} \mathrm{~s}^{-1}$, with a period of $\sim 1.5$ days. Clearly, in the majority of cases, the rotational velocity modulation will be smaller than the orbital modulation induced by the BD. In general, our task will simply be to deconvolve the two radial velocity components. This should generally be easy. For synchronized orbits, one could measure additional radial velocity data at a later date, when the spot modulation has changed.

\section{CONCLUSIONS}

In order to assess the prospects of discovering eclipsing BD companions capable of yielding mass, age, radius, metallicity, and luminosity data, we have assessed what is currently known about such systems. Although close BD companions are fairly rare around late $\mathrm{K}$ and early-to-mid $\mathrm{M}$ dwarfs, there could be a $\sim 1 \%$ companion fraction at very close $(\sim 0.01-0.02$ AU) separation. Our knowledge of this companion fraction is poor, but if correct, it suggests that although the BD desert extends into this regime, it is not as "dry" as for solar-type stars.

Using a simulation and the SuperWASP project as an example, we have predicted the numbers and properties of close BD companions that could be identified by ultra-wide-field transit surveys in the next few years. We assumed a $1 \%$ close $\mathrm{BD}$ companion fraction at $0.02 \mathrm{AU}$, a uniform age range from 0 to $10 \mathrm{Gyr}$, and a BD mass function of $\alpha=0.5$. We estimate SuperWASP to be sensitive to transits around $\sim 1$ million late $\mathrm{K}$ and early-to-mid M dwarfs. Based on our current knowledge of BD properties, our simulation predicts that $\sim 400 \mathrm{BDs}$ in eclipsing binaries could be found. It predicts that $\sim 60$ of these binaries should have secondary eclipses that are measurable from follow-up NIR light curves and should thus provide direct measurement of the BD brightness. The most common detectable BDs are expected to be higher mass BDs orbiting late $\mathrm{K}$ to $\mathrm{M} 3$ primaries. The majority $(\sim 70 \%)$ of the detectable BDs should not be irradiated by more than $10 \%$.

The simulation also reveals a preference for youth among binaries containing detectable BDs. This results from the fact that younger BDs are brighter relative to their primary. Our simulation predicts that $\sim 20$ binaries containing detectable BDs will have ages under $1 \mathrm{Gyr}$. We suggest that it is likely that a significant fraction of such young systems could be young moving-group members: disk populations with characteristic space motions that are believed to share a common origin and thus have an age that can be well constrained (see $\S 5$ ). The typical spectral type of the BDs in this young subsample ranges from M5 to L5 and may be bimodal, peaking at M5-M9 and L3L5. Higher mass BDs are still preferred, but the preference is not as strong for the young subset, and the population is more evenly spread over the mass range $0.040-0.075 M_{\odot}$. We expect $\sim 80 \%$ of these young companion BDs not to be irradiated by more than $10 \%$.

Although some of our input assumptions are rather uncertain, we show that transit surveys such as SuperWASP could find significant numbers of eclipsing BD companions, and by carrying out the follow-up measurements we describe, it would be possible to accurately determine mass, age, radius, metallicity, and luminosity data for $\sim 10-20$ young ( $<1$ Gyr age) BDs. The majority of these $(\sim 80 \%)$ should not be irradiated above the $10 \%$ level and could thus provide an empirical test bed to compare to theoretical models of free-floating BDs.

We thank Don Pollacco, Francis Keenan, Pete Wheatley, Richard West, and Simon Hodgkin for useful discussions. The authors are grateful to PPARC for their support of this work.

\section{REFERENCES}

Alonso, R., et al. 2004, ApJ, 613, L153

Armitage, P. J., \& Bonnell, I. A. 2002, MNRAS, 330, L11

Asiain, R., Figueras, F., \& Torra, J. 1999a, A\&A, 350, 434

Asiain, R., Figueras, F., Torra, J., \& Chen, B. 1999b, A\&A, 341, 427

Bakos, G., Noyes, R. W., Kovács, G., Stanek, K. Z., Sasselov, D. D., \& Domsa, I. 2004, PASP, 116, 266

Baraffe, I., Chabrier, G., Allard, F., \& Hauschildt, P. H. 1998, A\&A, 337, 403

Benn, C. R., \& Ellison, S. 1998, NewA Rev., 42, 503

Bessell, M. S. 1990, PASP, 102, 1181

Blundell, J., et al. 2003, MNRAS, submitted

Bouy, H., et al. 2004, A\&A, 423, 341

Burgasser, A. J., Kirkpatrick, J. D., Reid, I. N., Brown, M. E., Miskey,

C. L., \& Gizis, J. E., 2003, ApJ, 586, 512
Burrows, A., Sudarsky, D., \& Hubbard, W. B. 2003, ApJ, 594, 545

Caldwell, D. A., et al. 2004, in IAU Symp. 213, Bioastronomy 2002: Life among the Stars, ed. R. Norris \& F. Stootman (San Francisco: ASP), 93

Castro, S., Porto de Mello, G. F., \& da Silva, L. 1999, MNRAS, 305, 693

Chabrier, G., Baraffe, I., Allard, F., \& Hauschildt, P. 2000, ApJ, 542, L464

Chabrier, G., Barman, T., Baraffe, I., Allard, F., \& Hauschildt, P. H. 2004, ApJ, 603, 53

Charbonneau, D., Brown, T. M., Latham, D. W., \& Mayor, M., 2000, ApJ, 529, L45

Christian, D. J., Craig, N., Dupuis, J., Roberts, B. A., \& Malina, R. F. 2001, AJ, 122, 378 
Christian, D. J., et al. 2004, in ESO Conf. Proc., Cool Stars 13, in press

Close, L. M., Siegler, N., Freed, M., \& Biller, B. 2003a, ApJ, 587, 407

Close, L. M., et al. 2003b, ApJ, 599, 537

Dahn, C. C., et al. 2002, AJ, 124, 1170

Dehnen, W. 1998, AJ, 115, 2384

De Simone, R., Wu, X., \& Tremaine, S. 2004, MNRAS, 350, 627

Doyle, J. G., Houdebine, E. R., Mathioudakis, M., \& Panagi, P. M. 1994, A\&A, 285, 233

Dravins, D., Lindegren, L., Mezey, E., \& Young, A. T. 1998, PASP, 110,610

Dreizler, S., Rauch, T., Hauschildt, P., Schuh, S. L., Kley, W., \& Werner, K. 2002, A\&A, 391, L17

Duquennoy, A., \& Mayor, M. 1991, A\&A, 248, 485

Els, S. G., Sterzik, M. F., Marchis, F., Pantin, E., Endl, M., \& Kürster, M. 2001, A\&A, 370, L1

Fischer, D. A., \& Marcy, G. W. 1992, ApJ, 396, 178

Freed, M., Close, L. M., \& Siegler, N. 2003, in IAU Symp. 211, Brown Dwarfs, ed. E. Martín (San Francisco: ASP), 261

Gizis, J. E., Kirkpatrick, J. D., Burgasser, A., Reid, I. N., Monet, D. G., Liebert, J., \& Wilson, J. C. 2001, ApJ, 551, L163

Gizis, J. E., Reid, I. N., \& Hawley, S. L. 2002, AJ, 123, 3356

Halbwachs, J. L., Arenou, F., Mayor, M., Udry, S., \& Queloz, D. 2000, A\&A, 355, 581

Halbwachs, J. L., Mayor, M., Udry, S., \& Arenou, F. 2003, A\&A, 397,159

Hawley, S. L., Reid, I. N., \& Tourtellot, J. G. 1999, Very Low Mass Stars and Brown Dwarfs, ed. R. Rebolo \& M. R. Zapatero Osorio (Cambridge: Cambridge Univ. Press), 109

Hayes, D. S. 1985, in IAU Symp. 111, Calibration of Fundamental Stellar Quantities (Dordrecht: Reidel), 225

Henry, G. W., Baliunas, S. L., Donahue, R. A., Fekel, F. C., \& Soon, W. 2000a, ApJ, 531, 415

Henry, G. W., Marcy, G. W., Butler, R. P., \& Vogt, S. S. 2000b, ApJ, $529, \mathrm{~L} 41$

Henry, T. J., \& McCarthy D. J., Jr. 1993, AJ, 106, 773

Henry, T. J., Soderblom, D. R., Donahue, R. A., \& Baliunas, S. L. 1996, AJ, 111, 439

Horne, K. 2003, in ASP Conf. Ser. 294, Scientific Frontiers in Research on Extrasolar Planets, ed. D. Deming \& S. Seager (San Francisco: ASP), 387

Jao, W.-C., Henry, T. J., Subasavage, J. P., Bean, J. L., Costa, E., Ianna, P. A., \& Mendez, R. A. 2003, AJ, 125, 332

Jeffries, R. D., Oliveira, J. M., Barrado y Navascués, D., \& Stauffer, J. R. 2003, MNRAS, 343, 1271

Kane, S. R., et al. 2004, MNRAS, 353, 689

Kidger, M. R., \& Martín-Luis, F. 2003, AJ, 125, 3311

King, J. R., Villarreal, A. R., Soderblom, D. R., Gulliver, A. F., \& Adelman, S. J. 2003, AJ, 125, 1980

Knapp, G. R., et al. 2004, AJ, 127, 3553

Konacki, M., Torres, G., Sasselov, D. D., \& Jha, S. 2003, ApJ, 597, 1076

Kotoneva, E., Flynn, C., \& Jimenez, R. 2002, MNRAS, 335, 1147

Krishnamurthi, A., et al. 1998, ApJ, 493, 914

Kroupa, P., Aarseth, S., \& Hurley, J. 2001, MNRAS, 321, 699

Lachaume, R., Dominik, C., Lanz, T., \& Habing, H. J. 1999, A\&A, 348,897

Leggett, S. K., Allard, F., Dahn, C., Hauschildt, P. H., Kerr, T. H., \& Rayner, J. 2000, ApJ, 535, 965

Leggett, S. K., et al. 2002, ApJ, 564, 452

Luhman, K. L. 2004, ApJ, 614, L398

Lui, M. C., Fischer, D. A., Graham, J. R., Lloyd, J. P., Marcy, G. W., \& Butler, R. P. 2002, ApJ, 571, 519
Lyngå, G. 1987, Catalogue of Open Cluster Data (5th ed.; Strasbourg: CDS)

Makarov, V. V. 2003, AJ, 126, 1996

Makarov, V. V., \& Urban, S. 2000, MNRAS, 317, 289

Marchal, L., et al. 2003, Proc. IAU Symp. 211, Brown Dwarfs, ed. E. Martín (San Francisco: ASP), 311

Marcy, G. W., \& Benitz, K. J. 1989, ApJ, 344, 441

Mathioudakis, M., \& Doyle, J. G. 1991, A\&A, 244, 409

McCaughrean, M. J., et al. 2004, A\&A, 413, 1029

Mochnacki, S. W., et al. 2002, AJ, 124, 2868

Montes, D., López-Santiago, J., Gálvez, M. C., Fernandez-Figueroa, M. J., De Castro, E., \& Cornide, M., 2001, MNRAS, 328, 45

Moreno, E., Alfaro, E. J., \& Franco, J. 1999, ApJ, 522, 276

Mountain, C. M., Leggett, S. K., Selby, M. J., \& Zadrozny, A. 1985, A\&A, 150, 281

Nidever, D. L., Marcy, G. W., Butler, R. P., Fischer, D. A., \& Vogt, S. S. 2002, ApJS, 141, 503

Nordström, B., et al. 2004, A\&A, 418, 989

Odenkirchen, M., Soubiran, C., \& Colin, J. 1998, NewA, 3, 583

Oppenheimer, B. R., et al. 2001, AJ, 121, 2189

Pickles, A. J. 1985, ApJS, 59, 33

Pinfield, D. J., Dobbie, P. D., Jameson, R. F., Steele, I. A., Jones, H. R. A., \& Katsiyannis, A. C. 2003, MNRAS, 342, 1241

Preibisch, T., Guenther, E., \& Zinnecker, H. 2001, AJ, 121, 1040

Randich, S., Pasquini, L., \& Pallavicini, R. 2000, A\&A, 356, L25

Reid, I. N., \& Cruz, K. L. 2002, AJ, 123, 2806

Reid, I. N., Gizis, J. E., \& Hawley, S. L. 2002, AJ, 124, 2721

Reid, I. N., Gizis, J. E., Kirkpatrick, J. D., \& Koerner, D. W. 2001, AJ, 121, 489

Reid, I. N., \& Mahoney, S. 2000, MNRAS, 316, 827

Reid, I. N., et al. 1999, ApJ, 521, 613

Reiners, A. 2004, in Proc. the 13th Cool Stars Workshop, ESA Spec. Pub. Ser., in press

Reipurth, B., \& Clarke, C. 2001, AJ, 122, 432

Ribas, I. 2003, A\&A, 400, 297

Santos, N. C., et al. 2002, A\&A, 392, 215

Sarajedini, A., von Hippel, T., Kozhurina-Platais, V., \& Demarque, P. 1999, AJ, 118, 2894

Schuh, S. L., et al. 2003, A\&A, 410, 649

Short, C. I., \& Doyle, J. G. 1998, A\&A, 336, 613

Soderblom, D. R., Duncan, D. K., \& Johnson, D. R. H. 1991, ApJ, 375,722

Song, I., Zuckerman, B., \& Bessell, M. S. 2003, ApJS, 599, 342

Stauffer, J. R., Schultz, G., \& Kirkpatrick, J. D. 1998, ApJ, 499, L199

Stauffer, J. R., et al. 2003, AJ, 126, 833

Steinmetz, M. 2003, in ASP Conf. Ser. 298, GAIA Spectroscopy: Science and Technology, ed. U. Munari (San Francisco: ASP), 381

Terndrup, D. M., et al. 2000, AJ, 119, 1303

Udalski, A., et al. 2002a, Acta Astron., 52, 1

- 2002b, Acta Astron., 52, 115 2003, Acta Astron., 53, 133

Udry, S., Mayor, M., Naef, D., Pepe, F., Queloz, D., Santos, N. C., \& Burnet, M. 2002, A\&A, 390, 267

Vogt, S. S., Butler, R. P., Marcy, G. W., Fischer, D. A., Pourbaix, D., Apps, K., \& Laughlin, G. 2002, ApJ, 568, 352

Wilson, J. C., Kirkpatrick J. D., Gizis, J. E., Skrutskie, M. F., Monet, D. G., \& Houck, J. R. 2001, AJ, 122, 1989

Young, A., Skumanich, A., MacGregor, K. B., \& Temple, S. 1990, ApJ, 349, 608

Zapatero Osorio, M. R., et al. 2002, A\&A, 384, 937

Zheng, Z., Flynn, C., Gould, A., Bahcall, J. N., \& Salim, S. 2001, ApJ, 555, 393 